\title{
Identification of human long noncoding RNAs associated with nonalcoholic fatty liver disease and metabolic homeostasis
}

\author{
Xiangbo Ruan,, ${ }^{1}$ Ping Li, ${ }^{1}$ Yonghe Ma, ${ }^{1}$ Cheng-fei Jiang, ${ }^{1}$ Yi Chen, ${ }^{1}$ Yu Shi, ${ }^{1}$ Nikhil Gupta, ${ }^{1}$ Fayaz Seifuddin, ${ }^{2}$ Mehdi Pirooznia, ${ }^{2}$ \\ Yasuyuki Ohnishi, ${ }^{3}$ Nao Yoneda, ${ }^{3}$ Megumi Nishiwaki, ${ }^{3,4}$ Gabrijela Dumbovic, ${ }^{5}$ John L. Rinn, ${ }^{5}$ Yuichiro Higuchi, ${ }^{3}$ Kenji Kawai, ${ }^{6}$ \\ Hiroshi Suemizu, ${ }^{3}$ and Haiming Cao ${ }^{1}$ \\ 'Cardiovascular Branch, National Heart, Lung, and Blood Institute, NIH, Bethesda, Maryland, USA. 'Bioinformatics and Computational Biology, National Heart, Lung, and Blood Institute, NIH, Bethesda, Maryland, \\ USA. ${ }^{2}$ Laboratory Animal Research Department, Biomedical Research Laboratory, Central Institute for Experimental Animals, Kawasaki, Japan. ${ }^{4}$ Technical Service Department, CLEA Japan Inc., Shizuoka, Japan. \\ ${ }_{5}^{5}$ Department of Biochemistry and BioFrontiers, University of Colorado Boulder, Boulder, Colorado, USA. ${ }^{6}$ Department Pathology Analysis Center, Central Institute for Experimental Animals, Kawasaki, Japan.
}

A growing number of long noncoding RNAs (IncRNAs) have emerged as vital metabolic regulators. However, most human IncRNAs are nonconserved and highly tissue specific, vastly limiting our ability to identify human IncRNA metabolic regulators (hLMRs). In this study, we established a pipeline to identify putative hLMRs that are metabolically sensitive, disease relevant, and population applicable. We first progressively processed multilevel human transcriptome data to select liver IncRNAs that exhibit highly dynamic expression in the general population, show differential expression in a nonalcoholic fatty liver disease (NAFLD) population, and respond to dietary intervention in a small NAFLD cohort. We then experimentally demonstrated the responsiveness of selected hepatic IncRNAs to defined metabolic milieus in a liver-specific humanized mouse model. Furthermore, by extracting a concise list of protein-coding genes that are persistently correlated with IncRNAs in general and NAFLD populations, we predicted the specific function for each hLMR. Using gain- and loss-offunction approaches in humanized mice as well as ectopic expression in conventional mice, we validated the regulatory role of one nonconserved hLMR in cholesterol metabolism by coordinating with an RNA-binding protein, PTBP1, to modulate the transcription of cholesterol synthesis genes. Our work overcame the heterogeneity intrinsic to human data to enable the efficient identification and functional definition of disease-relevant human IncRNAs in metabolic homeostasis.

\section{Introduction}

In the past 2 decades, metabolic diseases have reached epidemic proportions globally $(1,2)$, underscoring the urgent need for a better understanding of metabolism regulation in humans. Although significant progress has been made in mapping the makeup and wiring diagram of metabolic pathways in recent years, substantial gaps remain in our understanding of the underlying pathophysiology of major metabolic diseases, such as obesity, nonalcoholic fatty liver disease (NAFLD), and type 2 diabetes. Given that maintenance of metabolic homeostasis requires high-level coordination at the cellular, organ, and organismal levels (3-5), such a level of complexity often cannot be adequately modeled by cultured cells and needs to be studied in in vivo systems, such as mice, which have the advantage of being conducive to genetic manipulation. Although these animal-based experimental studies are essential to capture mechanistic and physiologically relevant understanding of the role of metabolism in health and disease, there are limitations

Authorship note: XR, PL, YM, and CFJ contributed equally to this work. Conflict of interest: The authors have declared that no conflict of interest exists. Copyright: ( 2021, American Society for Clinical Investigation.

Submitted: January 10, 2020; Accepted: October 9, 2020; Published: January 4, 2021

Reference information: / Clin Invest. 2021;131(1):e136336.

https://doi.org/10.1172/JCl136336. due to species differences and imperfect disease models. These species-distinct differences are further underscored by our incomplete understanding of the human genome, particularly the enormous landscape of noncoding regions. Because $2 \%$ of the human genome is sufficient to encode all protein-coding genes, the vast majority of the genome is noncoding and was once considered to be made of gene deserts. It is now well-established that most of the noncoding regions can be transcribed, giving rise to approximately 60,000 long noncoding RNAs (lncRNAs) (6), which would equate to 3 times the number of protein-coding genes. Growing evidence supports the notion that lncRNAs play a regulatory role in systemic energy metabolism in mice. For example, we have shown that a liver-enriched lncRNA, lncLSTR, regulates systemic lipid metabolism (7), and a second lncRNA, lncLGR, regulates glycogen content in mice (8). Robust mouse lncRNA metabolic regulators (mLMRs), such as Lexis, Mexis, and Blnc1 (9-11), have also been reported by many groups, and this list continues to expand. Furthermore, several genomewide transcriptome analyses in mice have identified hundreds of potential mLMRs in key metabolic organs, suggesting that mouse lncRNAs constitute an additional dimension of metabolic regulation $(12,13)$. If human IncRNAs exercise a similar function, studying their metabolic function could help systemically uncover novel regulatory mechanisms of human metabolism and expand our understanding of how metabolic disease is initiated and progresses. 
Despite their enormous potential, it is currently extremely difficult to define human lncRNA metabolic regulators (hLMRs), in part because of the multitude of challenges in assigning functions to lncRNAs in general, and in determining the metabolic function of human lncRNAs in particular. Current knowledge and technology limit our ability to identify and characterize lncRNA functions, especially relative to the progress that has been made for protein-coding genes. Since our current understanding of the sequence-function relationship of lncRNAs is very poor, we cannot use sequence features, such as functional domains in protein-coding genes, to place lncRNAs in a biological context (14).

To address this challenge in the context of energy metabolism, considerable efforts have been devoted to identifying mLMRs by analyzing the regulatory information of a lncRNA in response to various conditions to inform its function. For example, we have developed a pipeline to identify mLMRs based on their regulations by multiple pathophysiologically representative metabolic conditions in mice (12). Sallam et al. identified 2 mLMRs of cholesterol metabolism based on their regulation by liver $\mathrm{X}$ receptor (LXR), a well-established transcription factor in cholesterol homeostasis $(10,11)$. The valuable information yielded by these extensive studies could in theory help identify hLMRs if lncRNAs were as conserved as mRNAs. Surprisingly and intriguingly, however, over $80 \%$ of human lncRNAs are not conserved $(15,16)$, and most human lncRNAs cannot be found in mice and vice versa. Thus, most human lncRNAs belong to a unique class of molecules whose regulatory information has to be directly derived from human studies. Although clinical RNA-Seq data of human metabolic tissues are emerging and accumulating, their numbers and the metabolic conditions under which they are collected are limited. More importantly, unlike data generated in inbred mouse strains under well-controlled experimental conditions, the gene expression levels in humans are significantly affected by genetic heterogeneity (17) and environmental factors. With so many complicating factors involved, it is evidently not a trivial task to retrieve hLMR signals from human data that truly reflect metabolic responses, or are metabolically sensitive, without losing their significance to the general population, and an approach that is specifically suitable for human lncRNAs is needed. Furthermore, for protein-coding genes, the definitive functional validation is routinely carried out in research animals, particularly in mice, by creating gain- or loss-of-function models. Given that most human lncRNAs are nonconserved, their physiological function cannot be directly studied in conventional mice, and an in vivo model is needed to experimentally characterize putative hLMRs in a physiologically relevant setting.

In order to leverage the accumulating clinical studies to understand the pathophysiological importance of lncRNAs in human metabolism, we have established an effective strategy to retrieve a list of broadly representative and metabolically sensitive human lncRNAs from human transcriptome data. We further refined our selection based on the regulation of these human lncRNAs by defined metabolic conditions in a humanized mouse model, and most importantly, experimentally defined the in vivo role of a nonconserved hLMR in cholesterol metabolism in the humanized mouse model.

\section{Results}

Identification of hLMRs. To take advantage of the currently available human data while overcoming its limitations, we combined a variety of human studies and a humanized mouse model to establish a practical platform to identify population-applicable, metabolically sensitive, and disease-relevant hLMRs (Figure 1). In order to maintain the broad representation of selected lncRNAs in the general population, we started our analysis with human liver RNA-Seq data from the Genotype-Tissue Expression (GTEx) project $(18,19)$. Although GTEx RNA-Seq data harbor valuable information for distinct tissue-relevant genes, the data lack deep clinical phenotyping for identifying differentially expressed genes linked to metabolic disease or therapy. Interestingly, information on gene expression variability or dynamics in a specific cell type or tissue has been utilized to infer potential roles in pathophysiology and diseases (20-22). We postulate that lncRNAs with high expression variability in key metabolic tissues such as the liver in the general population from GTEx could be potentially metabolically sensitive and disease relevant. Therefore, we determined the gene expression variability in the livers of individuals in GTEx. To extend the coverage for human IncRNAs, we have recently established an updated and comprehensive lncRNA database, lncRNA Knowledgebase (lncRNAKB) (23), which we used to map human lncRNAs throughout this study (see Methods). In total, the coefficients of variation for 16,906 genes, including 2665 lncRNAs expressed in the liver samples (Figure 2A), were calculated and ranked in 4 quartiles. We then assigned the protein-coding genes in the top and bottom quartile separately to the category of complex diseases using the DAVID gene functional annotation tool. As shown in Figure 2B, there was a significant enrichment of multiple disease categories in the top quartile of protein-coding genes from the liver, whereas very few were found in the bottom quartile. This result suggests that hepatic genes with high dynamic expression in the general human population might be conditionally responsive and susceptible to a variety of complex disease conditions, particularly cardiometabolic diseases (Figure 2B). Thus, the 943 IncRNAs included in the top quartile (Supplemental Table 1; supplemental material available online with this article; https://doi. org/10.1172/JCI136336DS1) could be metabolically sensitive and potentially function as human lncRNA regulators in the development of cardiometabolic diseases (Figure 1, identification step 1).

To determine the responsiveness of these dynamic lncRNAs to a specific metabolic disease condition, we examined their regulation in NAFLD. NAFLD is a metabolic disorder of high prevalence and is known to cause global changes in gene expression and metabolism in the liver. Specifically, we analyzed a large RNA-Seq data set composed of liver samples (total 139 samples) from a cross-sectional study of NAFLD (24) (Figure 3A and Supplemental Table 2) and found that 348 of 943 ( $37 \%)$ dynamically expressed lncRNAs were differentially expressed in the NAFLD populations, further supporting the notion that highly dynamic lncRNAs may have direct relevance to metabolic disease (Figure 1, Identification steps 1-2).

Although the 348 lncRNAs have potential implications for both general and disease populations, their observed changes in expression levels could be affected by genetic heterogeneity inherent to population-based human studies. To minimize such effects and further enrich lncRNAs whose expression levels are indeed 


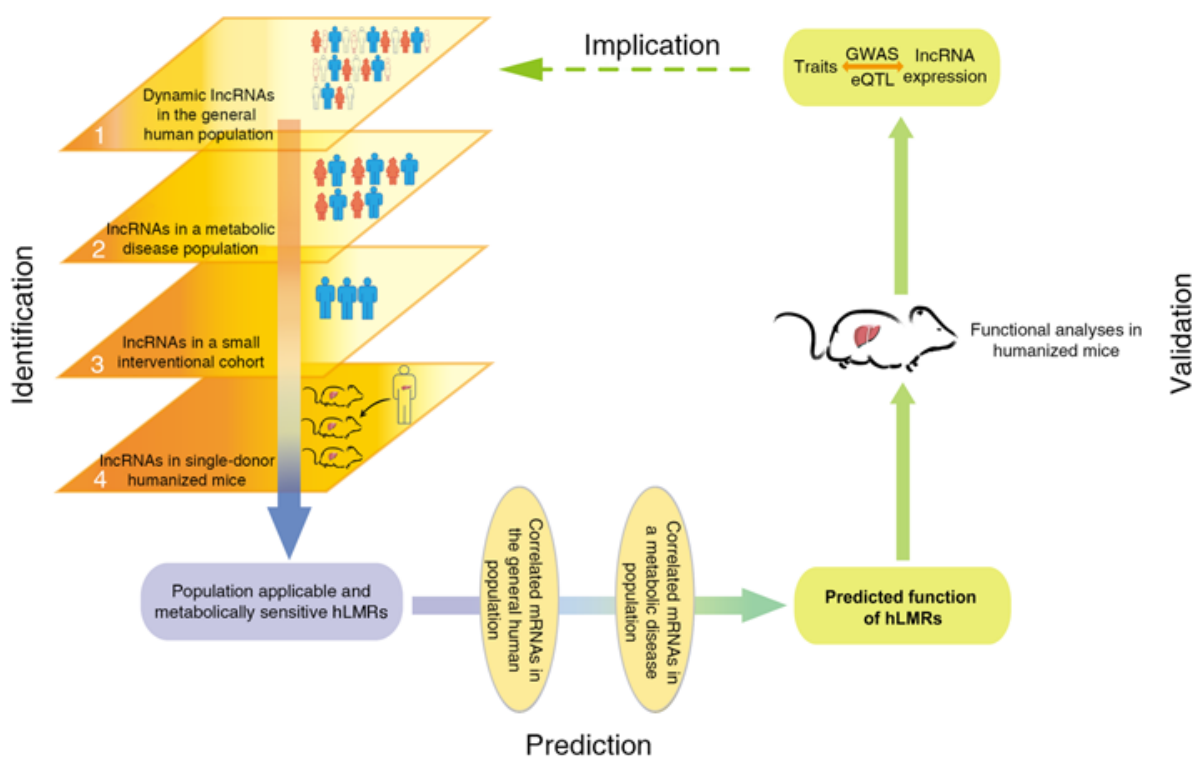

Figure 1. A roadmap for defining human IncRNA metabolic regulators. An integrative roadmap illustrates the steps to identify population-applicable, metabolically sensitive, and disease-relevant human IncRNA metabolic regulators by stepwise selections of human IncRNAs in the general population, a metabolic disease population, a small disease cohort, and humanized mice (identification); to infer their function based on their correlation with mRNAs in multiple populations (prediction); to validate their function in humanized mice (validation); and finally to explore their relevance to human diseases (implication). metabolically sensitive, we next analyzed a liver RNA-Seq data set generated from liver biopsies of 7 obese NAFLD patients before and after a short-term intervention with a low-carbohydrate diet (25). A low-carbohydrate diet is known to cause rapid and robust reductions of liver fat as well as changes in the expression of genes in multiple metabolic pathways (25). Out of the 348 lncRNAs, 77 were also regulated by the low-carbohydrate dietary intervention in this small cohort (Supplemental Table 3 and Supplemental Table 4 and Figure 3A). Thus, by integrating gene expression dynamics in the general population and gene regulation in observational and interventional human studies related to NAFLD, we effectively identified a group of population-applicable, metabolically sensitive, and disease-relevant human lncRNAs, which we refer to as putative hLMRs (Figure 1, identification steps 1-3).

In recent years, it has become a common practice to predict a lncRNA's function based on its correlated protein-coding genes in a large transcriptome data set $(12,26-28)$. On many occasions, however, the number of such correlated genes could be very large, and the predicted functions could be very broad. Additionally, the correlated genes often vary significantly in distinct populations. To overcome these limitations, we extracted genes persistently correlated with a lncRNA in multiple independent data sets, which we postulated could remove some protein-coding genes that were spuriously coregulated with a lncRNA and enrich the lncRNA's specifically correlated genes. Specifically, we intersected protein-coding genes correlated with a lncRNA in the general GTEx population and samples from the NAFLD study described above. As a result, we identified a concise list of correlated genes of each hLMR in a metabolic disease-relevant setting. The Gene Ontology (GO) analysis using these lists indicated that our identified hLMRs may function in diverse metabolic pathways, such as fatty acid oxidation, the cholesterol biosynthetic process, and glucose metabolism (Figure 3B and Supplemental Table 4).

Metabolic regulation of $h L M R s$ in humanized mice. After filtering out confounding variables to identify metabolically sensitive hLMRs and predict their functions, we next asked whether we could further investigate their metabolic responses under a physiologically relevant and well-controlled experimental condition. Indeed, we recently found that a liver-specific humanized mouse model, which was produced by human hepatocytes from a single donor and kept in a defined environment in an animal facility (29), is suitable for studying the regulation of human-specific lncRNAs (30). We thus performed RNA-Seq analysis to identify differentially expressed human genes in the liver-specific humanized mice subjected to a fasting-refeeding regime, which involves the 2 extreme ends of caloric cycles and is known to regulate nearly all key metabolic genes in vivo (Supplemental Table 5). As expected, the expression of protein-coding genes involved in fatty acid oxidation and gluconeogenesis was upregulated by fasting and downregulated by refeeding, whereas genes in the lipogenesis pathway showed the opposite pattern (Figure 4A and Supplemental Figure 1A), supporting the proper response of human genes to nutrient and hormone levels in the humanized liver. Furthermore, we noticed that a significant portion of differentially expressed human genes during fasting and refeeding overlapped with those in the NAFLD and the low-carbohydrate dietary intervention analysis (Figure 4B). These results further support the notion that the humanized liver maintains a proper gene expression response to metabolic milieu as the human liver does. Finally, we found that 20 out of 77 of the liver hLMRs that we identified were regulated by feeding cycles in the humanized mice (Figure 4C). Indeed, the specific regulations of these 20 hLMRs were largely in line with their predicted function. For example, lnckb.38556, which is downregulated by fasting and recovered by refeeding, is predicted to function in biosynthesis of cholesterol.

Taken together, by performing stepwise selections of lncRNAs from multiple data sets representing the general population, disease populations, interventional studies, and well-controlled experiments in humanized mice, we established a list of potential hLMRs that are population applicable, metabolically sensitive, and disease relevant (Figure 1, identification steps 1-4). Furthermore, by extracting protein-coding genes that persistently correlated with lncRNAs in independent populations, we were able to generate a concise list of genes that could be utilized to infer the function of 


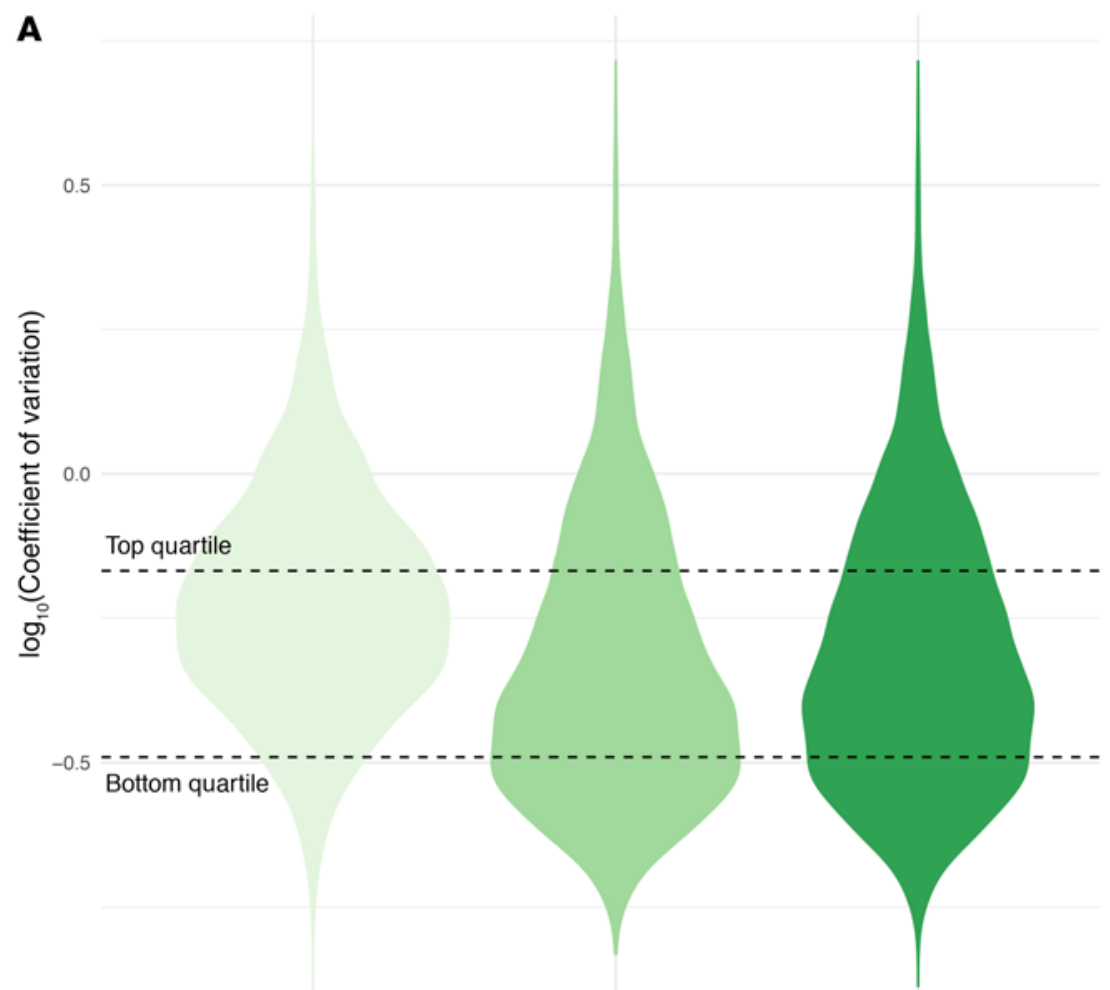

IncRNA genes Protein-coding genes Total

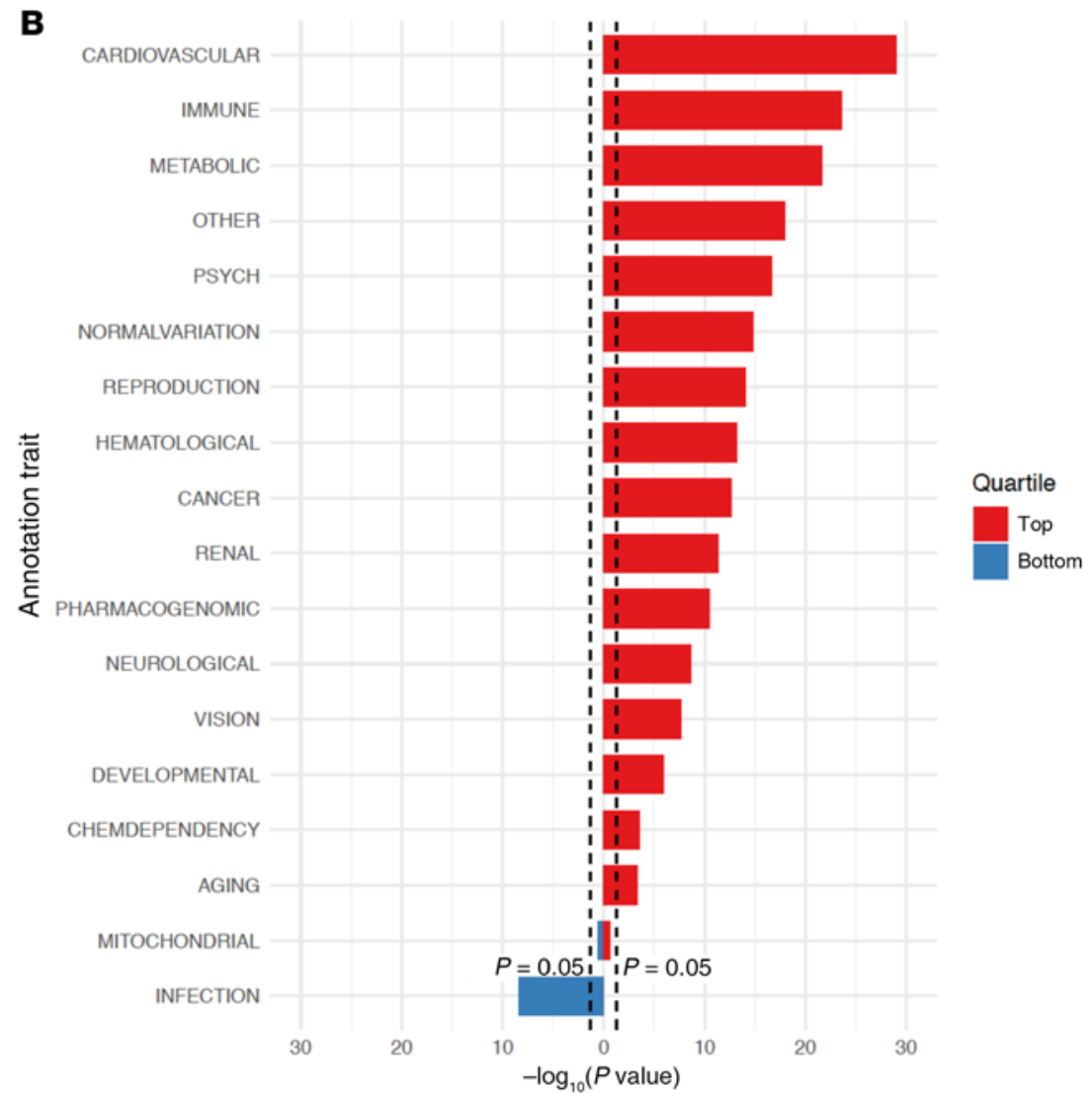

Figure 2. Gene expression variability in the livers of individuals in GTEx. (A) Violin plots of coefficient of variation of expressed genes $(n=16906)$ in GTEX liver data set ( $n=188$; scaled by area). Coefficient of variation was $\log _{10}$ normalized. (B) Enrichment of the disease classes assigned to the protein-coding genes in bottom quartile (left) and top quartile (right) dynamically expressed in GTEx liver data set.

each hepatic hLMR for downstream analysis (Figure 1, prediction steps).

Regulation of hepatic cholesterol biosynthetic pathway by hLMR1 in the humanized liver. Among the 20 liver hLMRs, we used lnckb.38556, which we refer to as hLMR1, as an example to experimentally validate our selection and prediction process. hLMR1 is annotated as a 5-exon intergenic lncRNA located in chromosome 3 of the human genome with the full-length transcript around $2 \mathrm{~kb}$, and bioinformatic prediction supported its poor coding potential (Supplemental Figure 1, B and C). No homolog of hLMR1 could be identified in mice by a BLAST search, suggesting it is a nonconserved human IncRNA. To further characterize the gene structure and transcript isoforms of hLMR1, we took advantage of the PacBio long-read RNA-Seq data (31) and found several hLMR1 isoforms with the same or very similar 5' end and exactly the same 3' end compared with the reference (Supplemental Figure 1D). Our cloned full-length hLMR1 included most exons detected in the PacBio isoforms, and an in vitro translation assay confirmed that this transcript was noncoding (Supplemental Figure 1, D and E). To avoid omission of functional isoforms of hLMR1, we designed our real-time PCR (qPCR) primers and shRNA targeting hLMR1 within its last exon, which was shared by all isoforms (Supplemental Figure 1D).

Our qPCR results using human tissue cDNA panels showed that hLMR1 was exclusively expressed in human liver tissue (Supplemental Figure 1F) and had a conservatively estimated copy number per cell of 56. Subcellular fractionation analysis using humanized liver tissues found that hLMR1 was distributed in the cytoplasm and nucleus, with more hLMR1 in the nuclear fraction (Supplemental Figure 2A). Single-molecule RNA (smRNA) FISH using cultured primary human hepatocytes further confirmed the predominantly nuclear localization of hLMR1 (Supplemental Figure 2B). RNASeq analysis of humanized livers showed that hLMR1 was downregulated during fasting and recovered upon refeeding (Figure 4C), which 
A
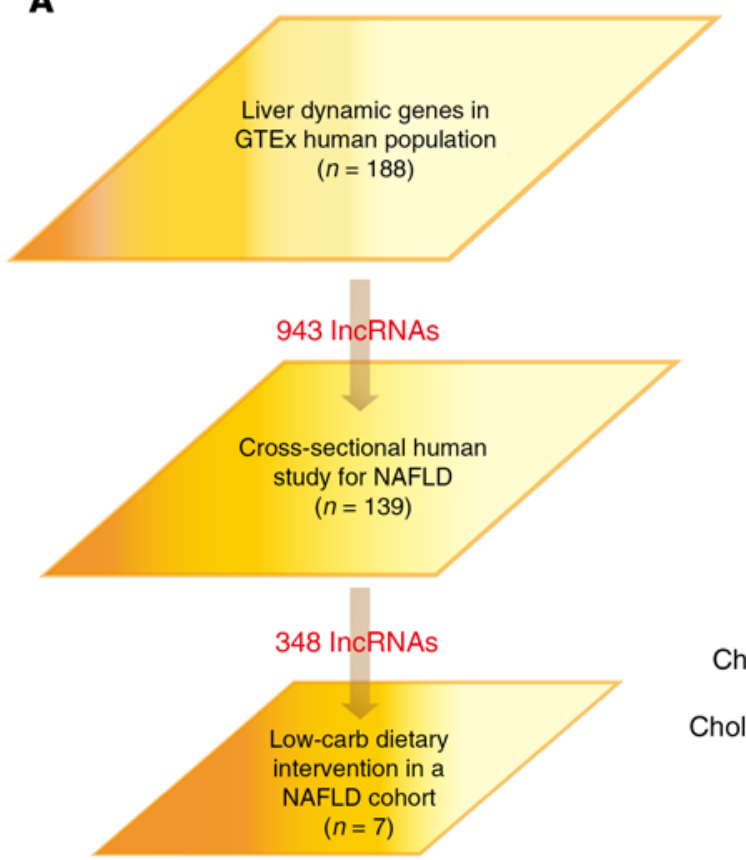

Figure 3. Identification of human IncRNA metabolic regulators. (A) The process of identification of human IncRNA metabolic regulators (hLMRs) by multilevel analysis of different types of relevant human data. The numbers of IncRNAs resulting from each step are marked in red. NAFLD, nonalcoholic fatty liver disease. (B) GO term analyses using the protein-coding genes persistently correlated with hLMRs in independent human populations. Only the top 5 GO terms of each hLMR are presented.
B

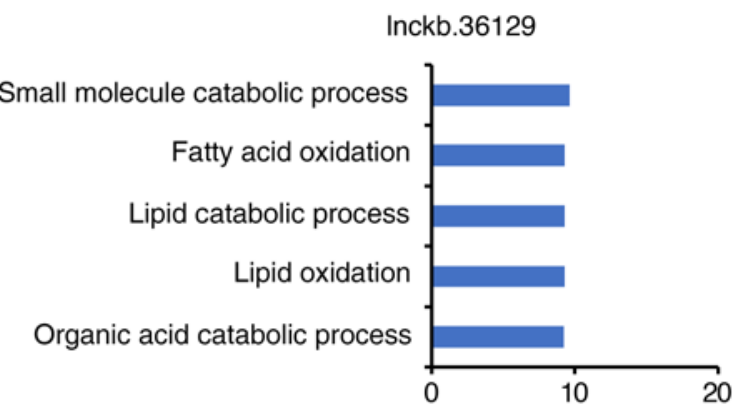

Inckb.38556 (hLMR1)

Cholesterol biosynthetic process via lathosterol

Cholesterol biosynthetic process via desmosterol

Isoprenoid biosynthetic process

Oxidation-reduction process

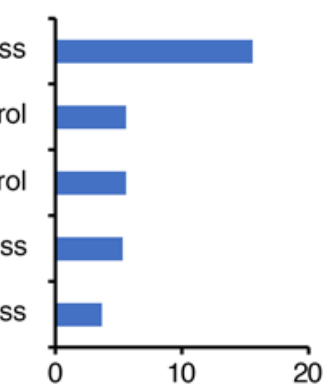

Inckb.12528

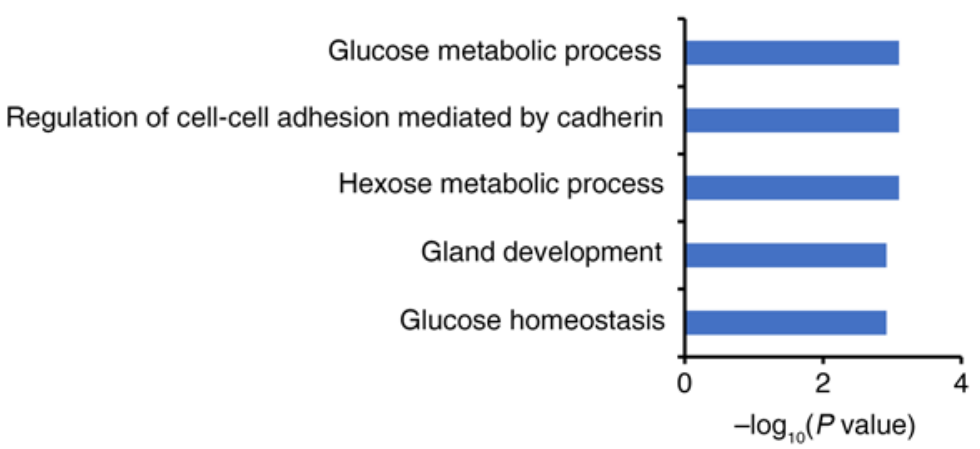

was further verified by qPCR (Supplemental Figure 2C). Furthermore, GO term analysis using the list of genes persistently correlated with hLMR1 indicated that hLMR1 may function in the cholesterol biosynthetic process (Figure 3B), which was consistent with the observation that hLMR1 was induced by refeeding.

To directly test the predicted role of hLMR1 in the cholesterol biosynthetic pathway, we screened for shRNAs that could efficiently block the expression of hLMR1, and then used adenoviruses expressing the selected shRNA to specifically reduce the expression levels of hLMR1. As shown in Figure 5A, this strategy successfully depleted the expression of hLMR1 in the livers of humanized mice, and a further subcellular fractionation analysis confirmed that both nuclear and cytosol hLMR1 could be efficiently reduced (Supplemental Figure 2D). Remarkably, among the 6 crucial genes in the cholesterol biosynthetic pathway whose expressions correlated with hLMR1 in our GO term analysis, 4 genes (SC5D, FDPS, LSS, and HMGCS1) showed decreased expression by more than $50 \%$ upon depletion of hLMR1 in the humanized livers (Figure 5A). This result thus supported the idea that hLMR1 positively regulates the cholesterol biosynthetic pathway, as predicted. We noticed that depletion of hLMR1 had no effect on the expression of PAQR9, the close neighbor gene of hLMR1 (Figure 5A), indicating it is unlikely that hLMR1 functions through modulating the expression of PAQR9.

Given that our pipeline was designed to identify hLMRs implicated in the general population, we next tested whether the regulatory effects of hLMR1 could be observed in a different genetic background. A knockdown experiment as described above was hence performed in humanized mice prepared with hepatocytes from a second independent and ethnically different donor. As shown in Figure 5B, with a similar knockdown efficiency of hLMR1 in these mice, we found significant downregulation of SC5D, LSS, FDPS, and HMGCS1, which was consistent with the result we observed in mice produced with the first donor. Taken together, our results indicate that hLMR1 is critical to maintain the expression of cholesterol biosynthetic genes in human populations.

To further study the regulatory effects of hLMR1, we next asked whether overexpression of hLMR1 could promote the expression of 


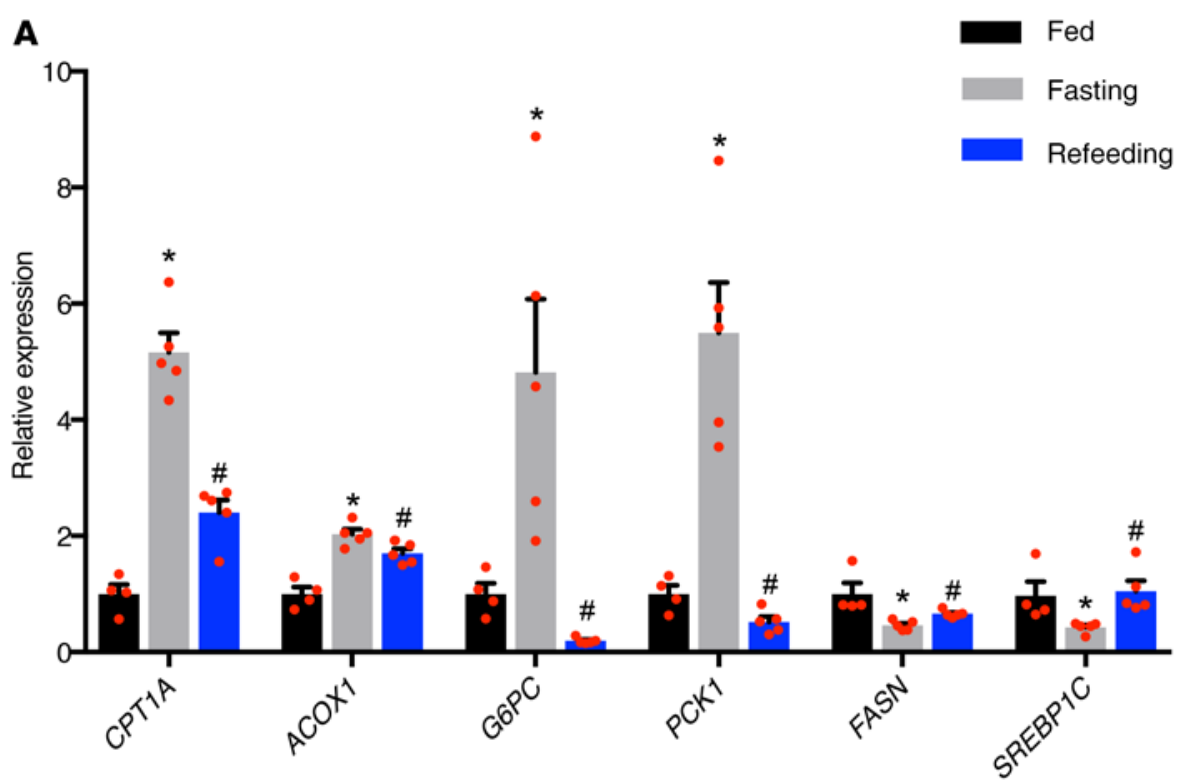

Figure 4. Metabolic regulation of human IncRNA metabolic regulators in humanized mice. (A) Gene expression in the livers from the humanized mice in response to fasting and refeeding (fed, $n=4$; fasting, $n=5$; refeeding, $n=5$ ), data are shown as the mean \pm SEM, ${ }^{*} P<0.05$ for fasting versus fed, ${ }^{\#} P<0.05$ for refeeding versus fasting by 2-tailed, unpaired Student's $t$ test. (B) Venn diagram for the intersection between metabolically responsive genes in humanized mice, the differentially expressed genes in the nonalcoholic fatty liver disease population (NAFLD), and the regulated genes by low-carbohydrate dietary intervention (LCD). (C) Heatmap of the relative mean expression levels for the human IncRNA metabolic regulators (hLMRs) in the livers from fed, fasted, and refed humanized mice. hLMR1 is marked by a red arrow.

B

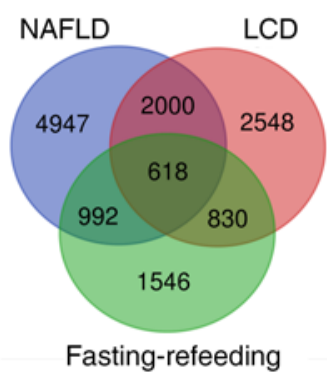

Fasting-refeeding
C

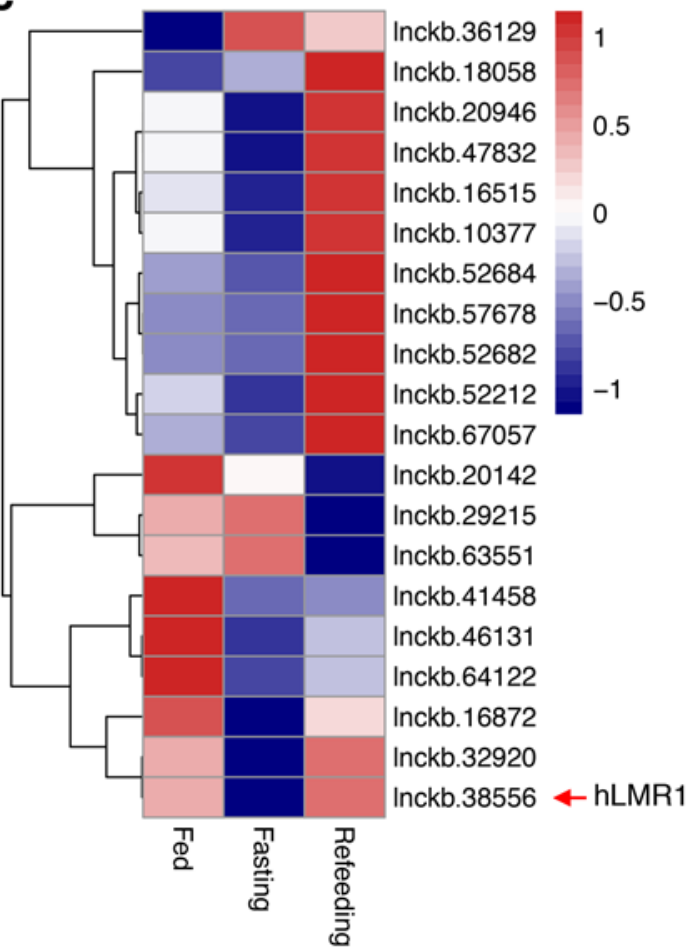

cholesterol levels in the humanized mice. The humanized livers in these mice are chimeric, and it is technically challenging to ascertain the specific impact of human hepatocytes. We thus used an immunoaffinity approach to specifically isolate human ApoB-containing lipoproteins from the plasma of humanized mice (Supplemental Figure 2E and Methods). Using this method, we found that depletion of hLMR1 in humanized livers led to a $40 \%$ decrease in human LDL and VLDL cholesterol levels compared with control humanized mice (Figure 5D). Taken together, our bioinformatic analyses using large-scale human data and functional analyses in humanized livers suggest that a nonconserved human lncRNA, hLMR1, is a crucial regulator of the hepatic cholesterol biosynthetic pathway and could play a critical role in the maintenance of cholesterol homeostasis in humans.

hLMR1 coordinates PTBP1 to promote the transcription of cholesterol biosynthetic genes. To explore the molecular mechanism mediating the regulatory effects of hLMR1, we first performed RNA poly II ChIP analysis to determine the transcriptional activities of hLMR1 target genes in the humanized livers. As shown in Figure 6A, humanized livers with depletion of hLMR1 showed significantly lower enrichment of RNA poly II on the transcription start site of human SC5D, FDPS, LSS, and HMGCS1. These data suggest that hLMR1 regulates the expression of cholesterol biosynthetic genes by promoting their transcription, in line with the relative enrichment of hLMR1 in the nuclear fraction (Supplemental Figure 2, A and B). To further explore how hLMR1 regulates the transcription of its target genes, we next performed RNA pulldown combined with mass spectrometry analysis to identify proteins that interact with hLMR1. This strategy successfully identified PTBP1, an RNA-binding protein that regulates almost all steps of 

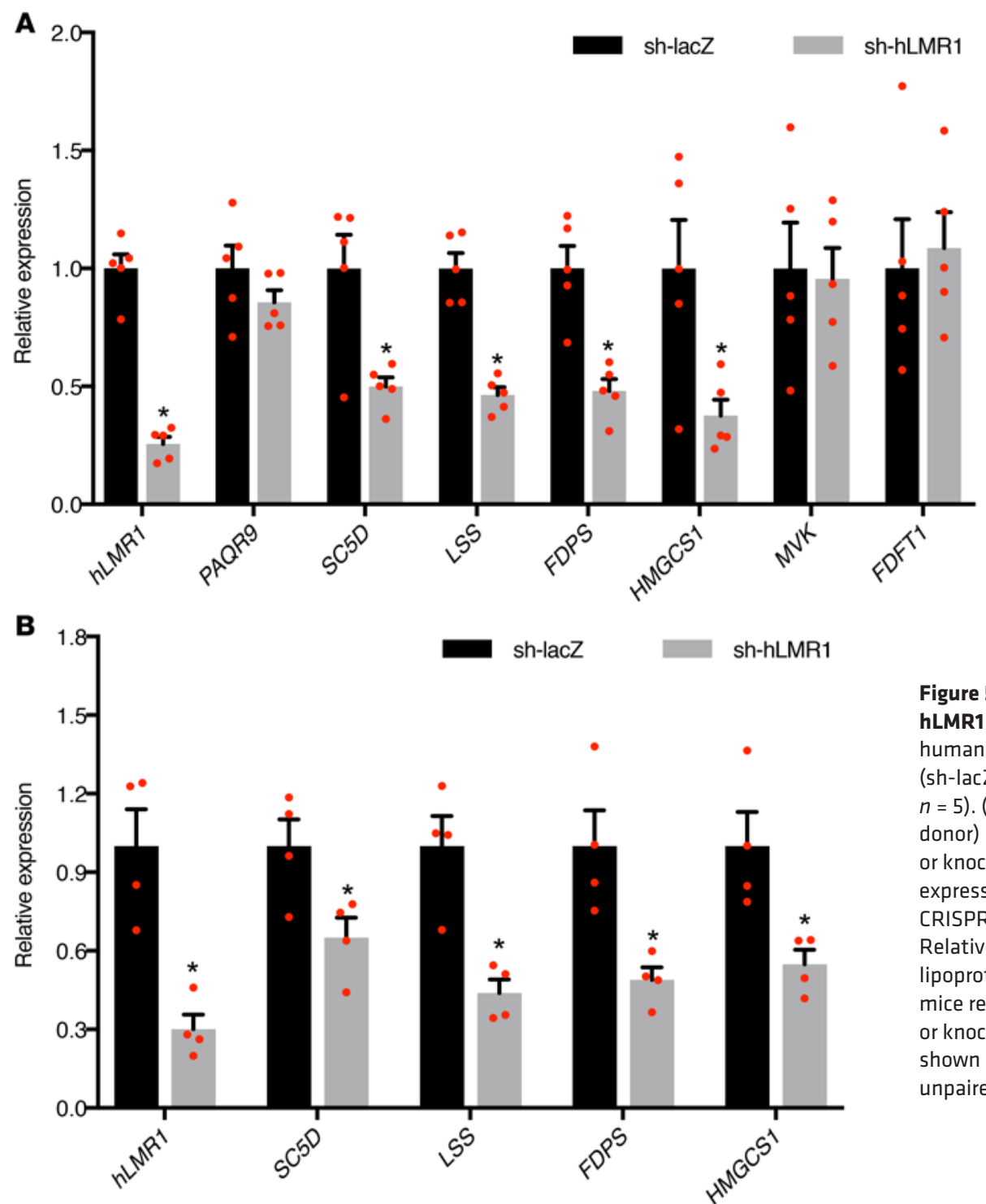

Figure 5. Regulation of cholesterol metabolism by hLMR1 in humanized mice. (A) Gene expression in humanized mice receiving adenovirus for control (sh-lacZ, $n=5$ ) or knockdown of hLMR1 (sh-hLMR1, $n=5$ ). (B) Gene expression in humanized mice (second donor) receiving adenovirus for control (sh-lac $Z, n=4)$ or knockdown of hLMR1 (sh-hLMR1, $n=4$ ). (C) Gene expression in humanized mice receiving adenovirus for CRISPRa-control $(n=4)$ or CRISPRa-hLMR1 $(n=3)$. (D) Relative cholesterol levels in human ApoB-containing lipoproteins purified from the plasma of humanized mice receiving adenovirus for control (sh-lacZ, $n=7$ ) or knockdown of hLMR1 (sh-hLMR1, $n=10$ ). Data are shown as the mean \pm SEM, ${ }^{*} P<0.05$ by 2 -tailed, unpaired Student's $t$ test.

C

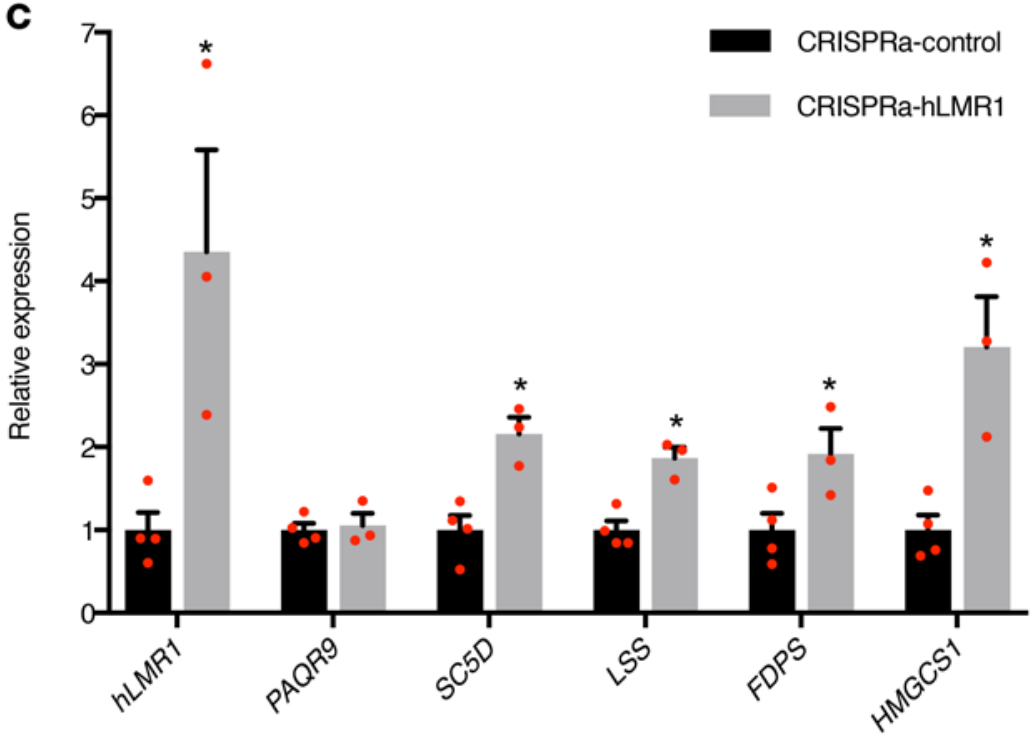

D

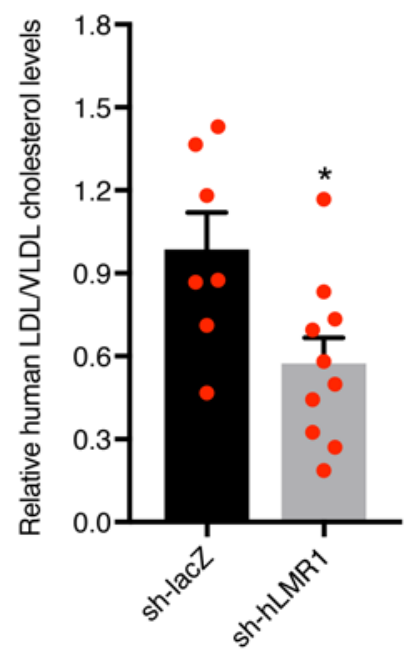




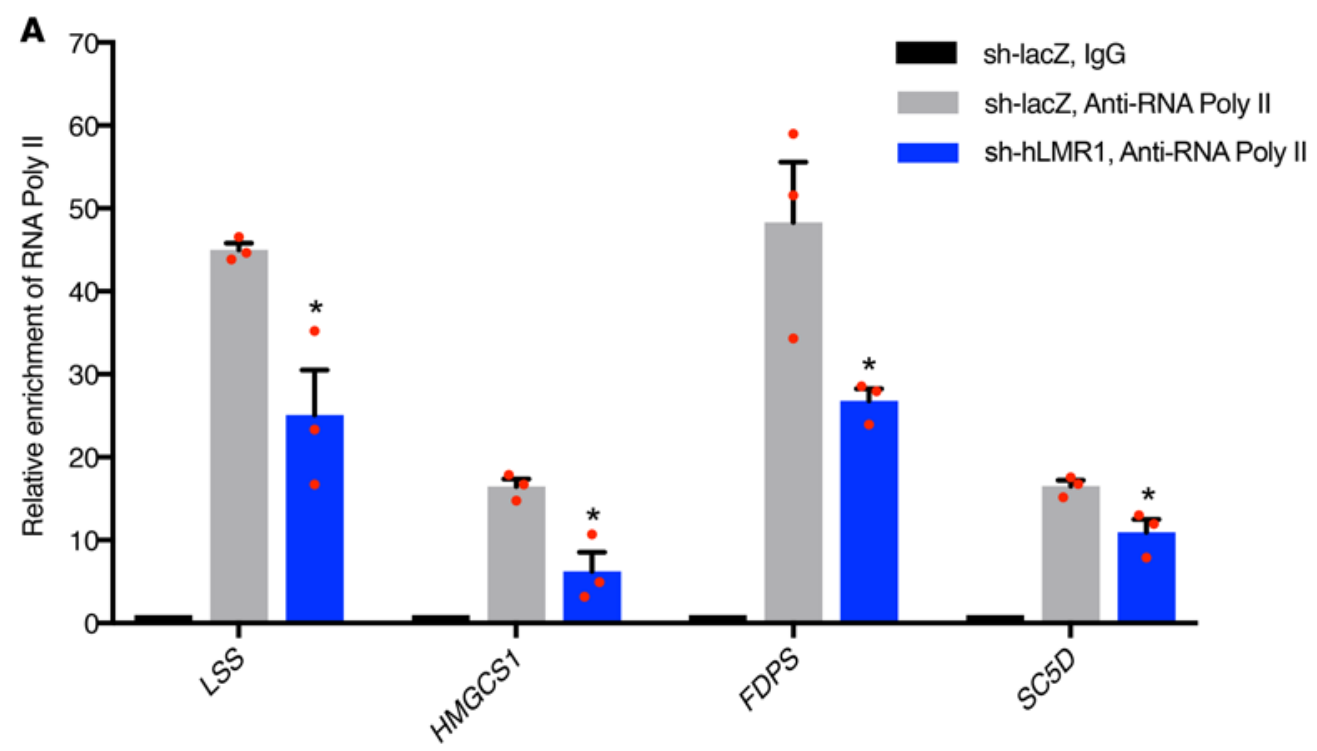

B

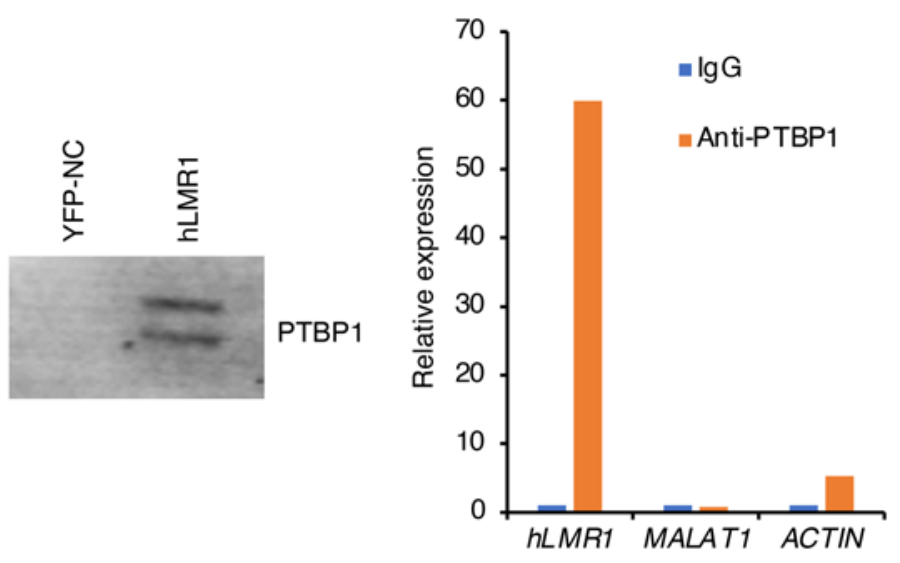

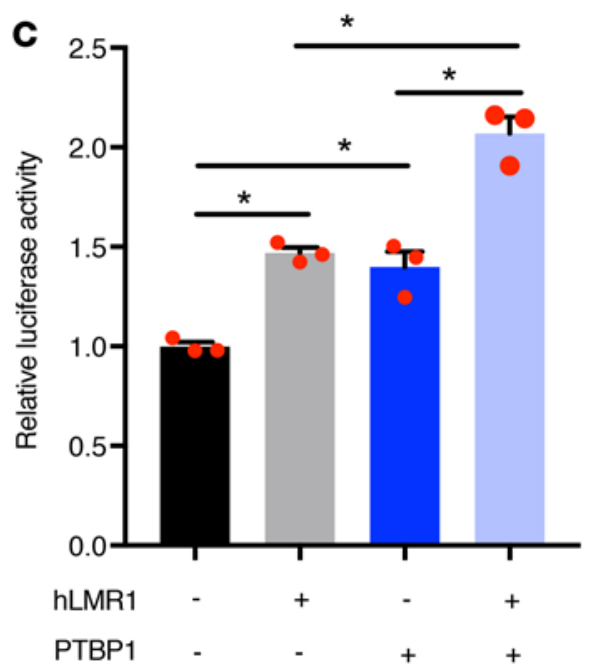

sh-lacZ, IgG

sh-lacZ, Anti-PTBP1

sh-hLMR1, Anti-PTBP1

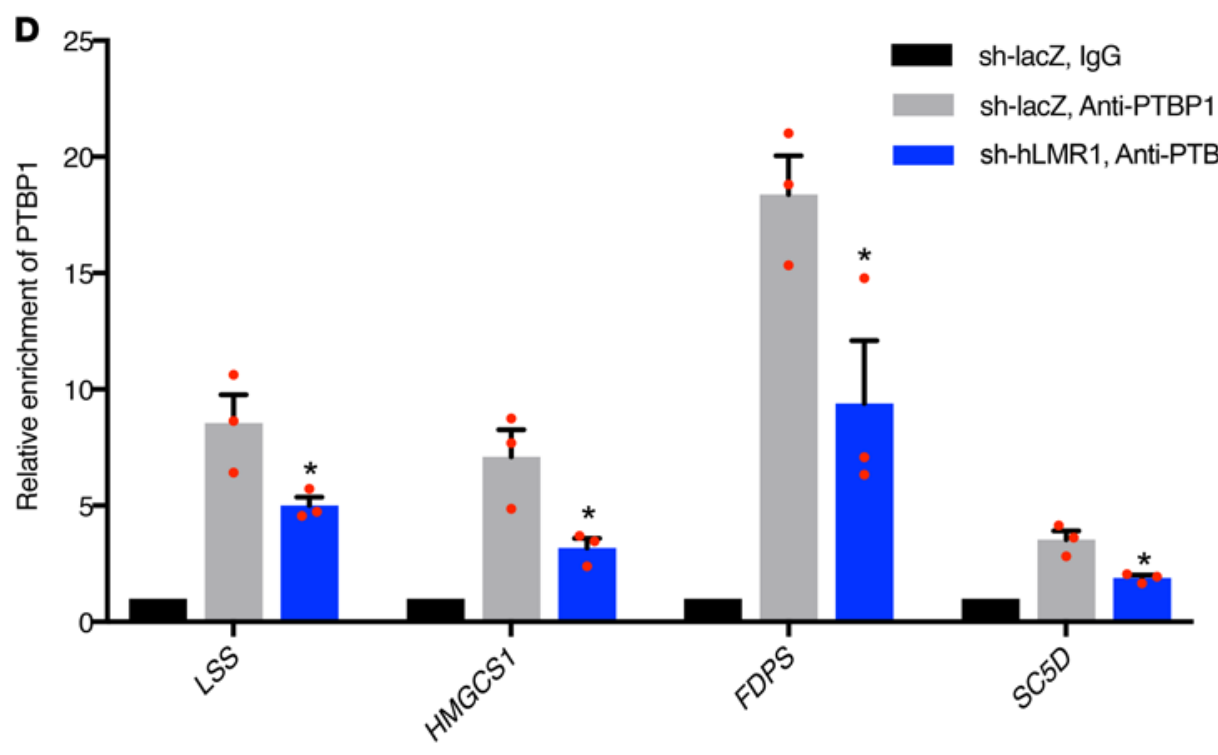

Figure 6. hLMR1 coordinates PTBP1 to promote the transcription of cholesterol biosynthetic genes. (A) RNA ploy II ChIP analyses in liver tissues of humanized mice receiving adenovirus for control (sh-lac $Z, n=3$ ) or knockdown of hLMR1 (sh-hLMR1, $n=3$ ). (B) Left: Western blot analysis of PTBP1 in hLMR1 pulldown, right: gene expression in PTBP1 RIP (RNA IP) in humanized liver. (C) HMCCS1 promoter-driven luciferase reporter assay in 293A cells ( $n=3$ for each group). Data are representative results of 3 independent experiments and are shown as the mean $\pm \mathrm{SEM},{ }^{*} P$ $<0.05$ by 1 -way ANOVA with post hoc Tukey's honestly significant difference test. (D) PTBP1 ChIP analyses in liver tissues of humanized mice receiving adenovirus for control (sh-lacZ, $n$ = 3) or knockdown of hLMR1 (sh-hLMR1, $n=3$ ). Data in $\mathbf{A}$ and $\mathbf{D}$ are shown as the mean \pm SEM, ${ }^{*} P<0.05$ by 2 -tailed, unpaired Student's $t$ test. 
RNA metabolism, as an hLMR1-binding partner. We confirmed the mass-spectrometry results by Western blot analysis (Figure 6B), and then an RNA IP (RIP) analysis using PTBP1 antibody in liver tissue lysate of humanized mice was performed. As shown in Figure 6B, hLMR1 was enriched by more than 50-fold in PTBP1 immunoprecipitate compared with IgG, further supporting the specific interaction between hLMR1 and PTBP1. To directly test whether PTBP1 is involved in hLMR1-mediated transcriptional regulation of cholesterol biosynthetic genes, we overexpressed hLMR1 in combination with PTBP1 to determine their effects on a human HMGCS1 promoter-driven luciferase reporter. As shown in Figure 6C, expression of either hLMR1 or PTBP1 could increase the HMGCS1 promoterdriven luciferase activity, and simultaneous expression of both showed a synergistic enhancing effect. This result suggests that PTBP1 is a positive transcriptional regulator of cholesterol biosynthetic genes, and hLMR1 likely functions through facilitating the recruitment of PTBP1 to the promoters of its target genes. To experimentally test this, we performed PTBP1 ChIP analysis in the humanized liver and determined PTBP1 enrichment on the promoters of hLMR1 target genes. As shown in Figure 6D, PTBP1 exhibited robust enrichment on the promoters of human SC5D, FDPS, LSS, and HMGCS1, and its binding to these promoters was diminished by depletion of hLMR1 in humanized livers. Taken together, our results indicate that hLMR1 recruited PTBP1 to the promoters of genes in the cholesterol biosynthesis pathway to activate their transcription. Considering the established role of PTBP1 in regulating pre-mRNA splicing, it is possible that hLMR1 could also affect the splicing of its target genes in addition to the transcriptional regulation. To test this possibility, we checked reported splicing events (32) as well as annotated splicing variants of hLMR1 target genes and found that the expression patterns of detected HMGCS1 and FDPS isoforms were not affected by knockdown of hLMR1 (Supplemental Figure 3, A-C), suggesting that it is unlikely that hLMR1 regulates the splicing of its target genes.

Ectopic expression of $h L M R 1$ in regular mice promotes cholesterol biosynthesis. To further study the functional importance of hLMR1PTBP1 in cholesterol metabolism, we asked whether the function of human PTBP1 is conserved in mice and as such, whether ectopic expression of hLMR1 in regular mice could promote cholesterol biosynthesis. To test these possibilities, we used adenovirus-mediated shRNAs to knock down Ptbp1 in regular mice and then examined the expressions of cholesterol biosynthetic genes. As shown in Figure 7A, when more than 70\% Ptbp1 was depleted, we observed decreased expression of $S c 5 d, F d p s$, Lss, and Hmgcs1. This result suggests that mouse Ptbp1 also positively regulates the expression of cholesterol biosynthetic genes, which allowed us to test the effect of ectopic expression of hLMR1 in regular mice. We thus cloned the full-length cDNA of hLMR1 in an adenoviral vector and delivered the packed adenoviruses into regular mice. This strategy successfully expressed hLMR1 in mouse livers to a level comparable to that in human hepatocytes. As shown in Figure 7B, we found that expression of hLMR1 resulted in marked upregulation of $S c 5 d, F d p s, L s s$, and Hmgcs 1 in the liver of regular mice but showed no effects on the expression of $S c d 1$ and $S p o t 14$, genes in the fatty acid biosynthesis pathway. These results suggest that as a humanspecific lncRNA, hLMR1 may function in the liver of regular mice through a similar mechanism as we observed in human experimental systems. To test this, we first determined whether hLMR1 could interact with the promoter regions of the mouse genes regulated by hLMR1. As shown in Supplemental Figure 4A, when incubated with liver chromatin lysates of regular mice, hLMR1 could enrich the promoter DNA fragments of $S c 5 d, L s s, F d p s$, and Hmgcs1. We further performed a PTBP1 ChIP analysis using liver chromatin lysates from regular mice with or without ectopic expression of hLMR1 and found that hLMR1 could enhance the binding of PTBP1 on the promoters of Sc5d, Lss, Fdps, and Hmgcs1 (Figure 7C). Furthermore, when Ptbp1 was depleted, ectopic expression of hLMR1 was no longer able to induce the expression of its target genes, suggesting that the regulatory effects of hLMR1 were dependent on the expression of Ptbp1 (Supplemental Figure 4B). Finally, both plasma and hepatic cholesterol levels were increased upon expression of hLMR1 (Supplemental Figure 4C). These data, in combination with our results in humanized mice, support the crucial role of hLMR1-PTBP1 complex in cholesterol metabolism.

Hepatic expression of hLMR1 is associated with cholesterol levels in human population. To further explore the impact of hLMR1 on cholesterol metabolism in the human population, we used expression quantitative trait loci (eQTL) and GWAS integrative analysis to determine the association between hepatic expression of hLMR1 and lipid levels in the general population (33). As shown in Figure 8, encouragingly, we found that several cis eQTLs of hLMR1 overlapped with GWAS loci for total cholesterol levels. A summary-data-based Mendelian randomization (SMR) analysis, which tests whether the effect size of an SNP on the phenotype is mediated by gene expression using data from GWAS and eQTL studies (34), was then performed to further determine whether the overlapped eQTL/GWAS loci were functionally related. The analysis passed both SMR and heterogeneity in dependent instruments (HEIDI) tests (SMR $P<0.05$ and HEIDI $P>$ 0.05 , see Methods), suggesting the hepatic expression level of hLMR1 might contribute to the regulation of cholesterol levels in humans.

\section{Discussion}

Human lncRNAs constitute a significant portion of the human transcriptome and have been shown to play a critical role in diverse biological processes. Despite this, the role of human lncRNAs in systemic energy metabolism is poorly understood, in part because of the challenging nature of identifying hLMRs and defining their metabolic function in a physiologically relevant context. In this work, we established an integrated bioinformatic and experimental pipeline to identify hLMRs based on their regulatory information in the general population, patients with metabolic disease, and a humanized mouse model (Figure 1). We also adapted an improved approach to infer the function of hLMRs. Finally, we provided a proof-of-principle example that the metabolic function of human lncRNAs could be successfully defined in a humanized mouse model, where we validated that a nonconserved human IncRNA exhibited its predicted function in cholesterol metabolism and confirmed it as a legitimate hLMR.

Compared to data generated in inbred mice under welldefined conditions, human gene expression data are intrinsically very noisy because of multiple confounding factors, particularly the diverse genetic background and environmental factors. On the other hand, steps to remove the impact of genetic and other confounding factors have the risk of restricting the significance of the findings to a specific subpopulation. To address these challenges, our work has established a platform to identify putative 


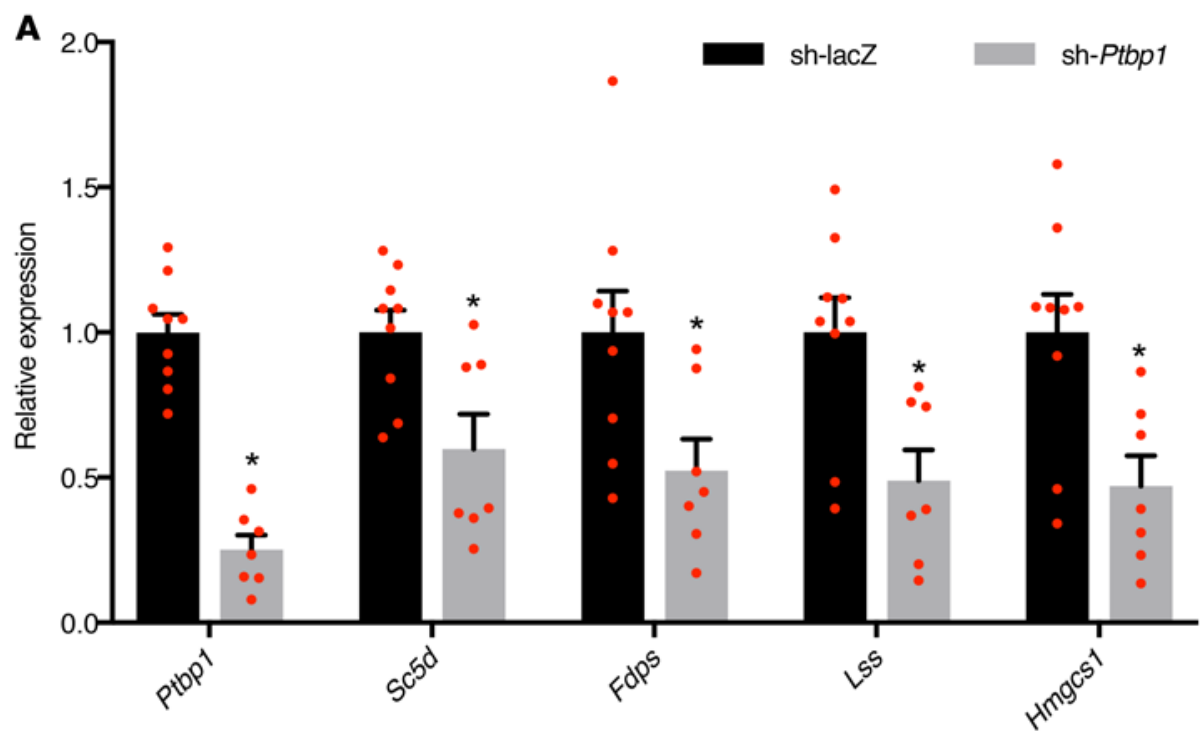

B
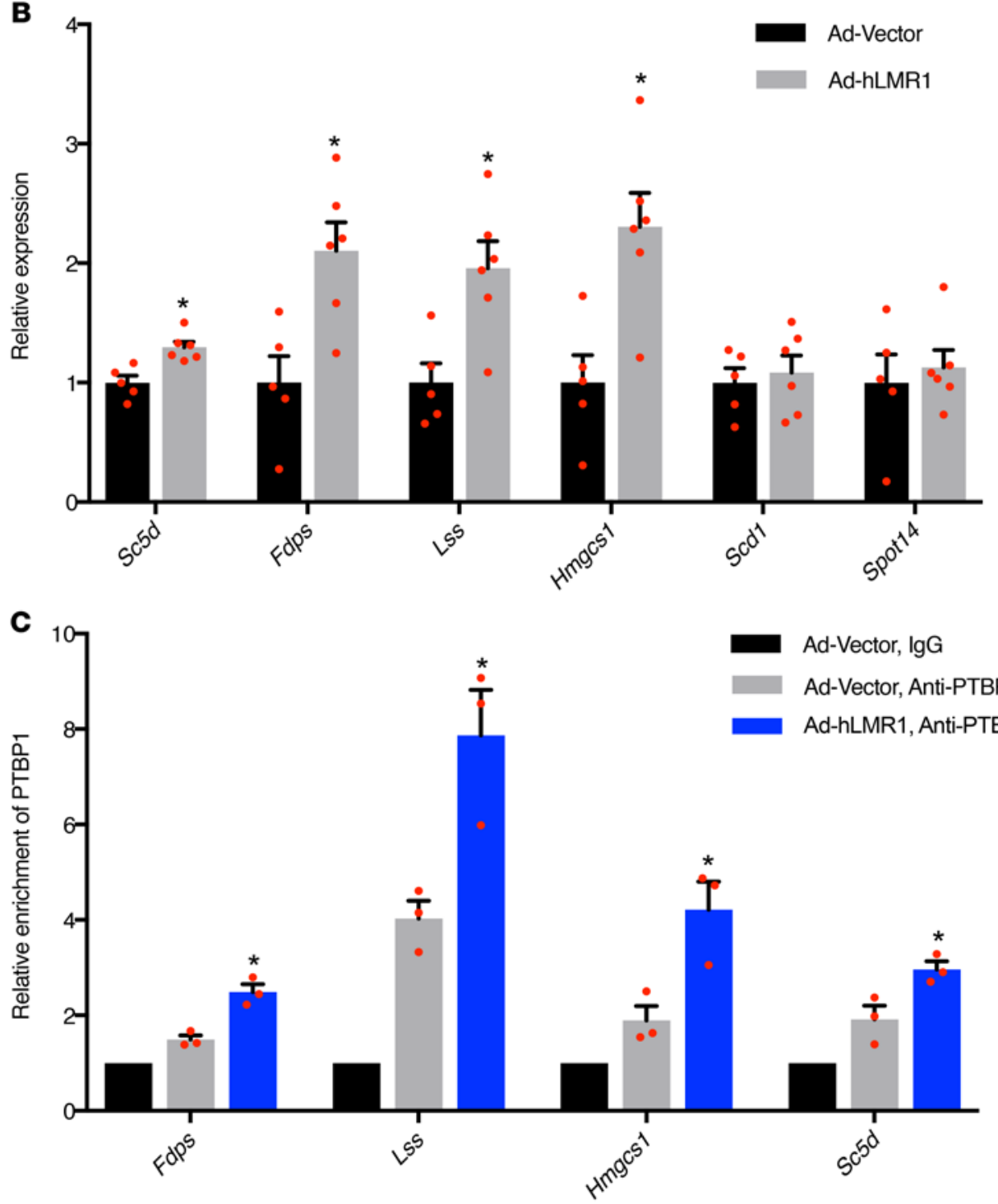

Figure 7. Ectopic expression of hLMR1 in regular mice promotes biosynthesis of cholesterol. (A) Gene expression in the livers of regular mice receiving lac $Z$ shRNA (sh-lacZ, $n=9$ ), or shRNA for Ptbp1 (sh-Ptbp1, $n=7$ ). (B) Gene expression in the livers of regular mice receiving adenovirus for control (ad-vector, $n$ =5) or expression of hLMR1 (ad-hLMR1, $n=6$ ). (C) PTBP1 ChIP analyses in liver tissues of regular mice receiving adenovirus for control (ad-vector) or expressing of hLMR1 (ad-hLMR1). $n=3$ for each group. Data in $\mathbf{A}, \mathbf{B}$, and $\mathbf{C}$ are shown as the mean \pm SEM, ${ }^{*} P<0.05$ by 2-tailed, unpaired Student's $t$ test.

hLMRs that are metabolically sensitive, disease relevant, and population applicable. Several steps in our selection procedure were designed to better retrieve specific signals against the background noises in human data and at the same time retain the significance of identified lncRNAs to the general population. For example, the responsiveness to multiple disease or metabolic conditions could enrich lncRNAs that are metabolically sensitive, and regulations in small cohorts and the general population alike could ensure that the functional importance of selected lncRNAs has broad implications.

Our approach is entirely scalable and can readily integrate any new RNA-Seq data of human metabolic organs under any additional metabolic conditions. These data are expected to become available en masse with the widespread use of RNA-Seq technology. Although our selective procedure could potentially enrich functional lncRNAs that play a role in metabolism, it does not immediately confirm functionality. Thus, this work also underscores the fundamental importance of a humanized mouse model for the definitive validation of the physiological importance of any putative hLMRs, which are mostly nonconserved. Expression of human lncRNAs in conventional mice, as we have utilized in the work, is a useful alternative to humanized mice, and it may mitigate some of the limitations 


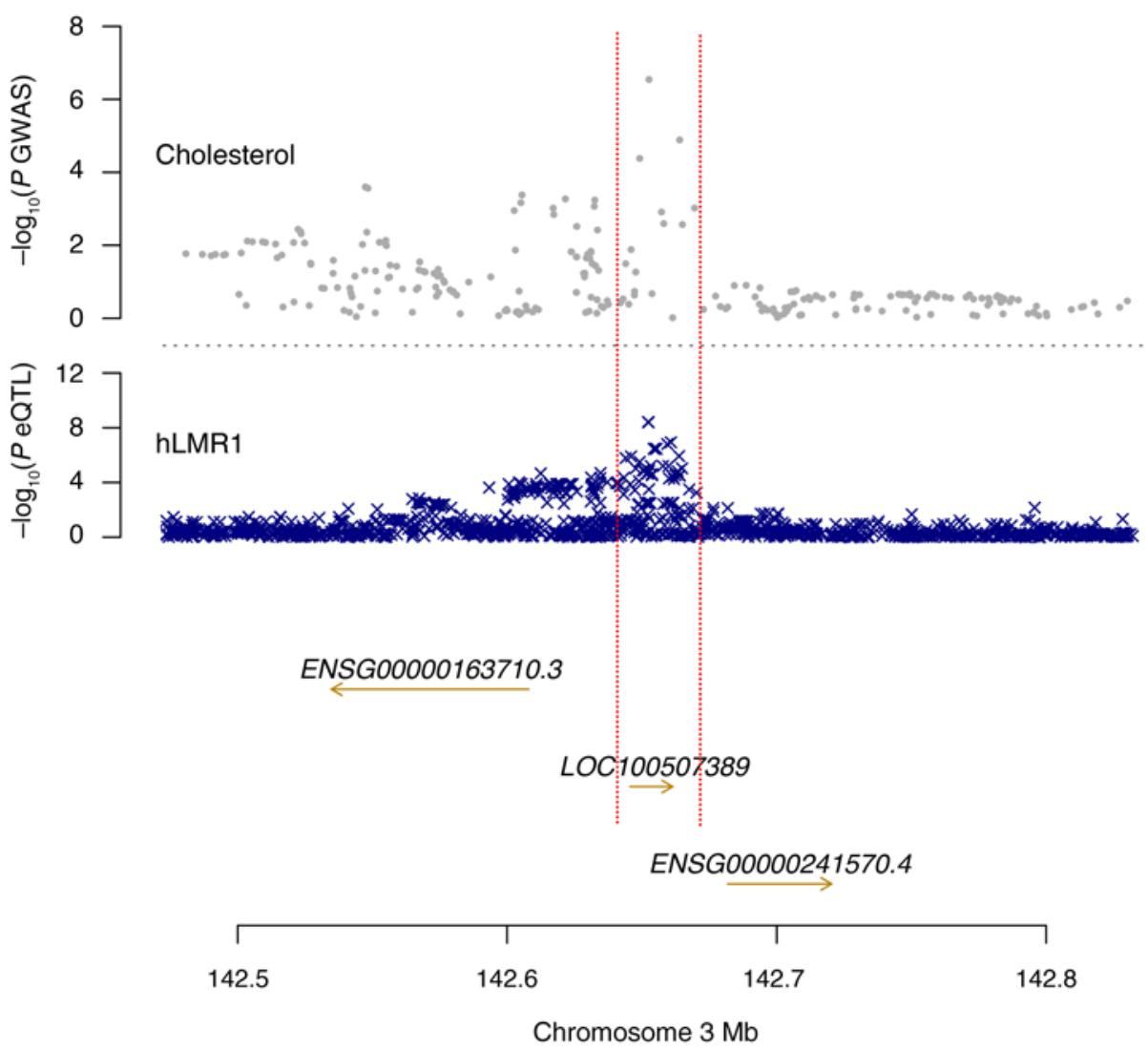

Figure 8. Illustration of the overlap between eQTLs of hLMR1 and GWAS loci for lipid trait. for how PTBP1 exercises its function in cells and animals. PTBP1 is a well-established regulator of splicing, but its identified functions have expanded rapidly in recent years. A number of lncRNAs have been recently reported to interact with PTBP1 to regulate its known functions or expand its functional space. For example, lncRNA LUCAT1 interacts with PTBP1 to facilitate alternative splicing of a set of DNA damage-related genes (37), and lncRNAs MEG3 and UCA1 have been shown to form a complex with PTBP1 to regulate RNA decay (38) or enhance RNA stability (39), respectively. Several lncRNAs have also been recently reported to bind to PTBP1 to regulate transcription $(40,41)$, which represents a relatively newly identified function for PTBP1. PTBP1 is universally and abundantly expressed in most tissues, but loss-offunction studies in animals have showed that it often exerts a tissue-specific function $(42,43)$, raising the question of how the tissue-specific regulation of $P T B P 1$ function is achieved. Compared to protein-coding genes, the expression of lncRNAs is much more cell type- and tissue-specific, and their specific interactions with PTBP1 might confer tissue of studying human lncRNAs in mice. However, ectopic expression of human lncRNAs in mice cannot replace the utility of humanized mice in which loss-of-function analysis of human lncRNAs provides a nearly definitive confirmation of their relevance to human physiology.

The function of hLMR1 identified in this study provides an interesting perspective on the regulation of human cholesterol synthesis genes. It is well known that humans and mice have very different lipoprotein profiles, largely due to the function of cholesterol ester transfer protein, a protein-coding gene that only exists in humans (not in mice) and can mediate the transfer of triglycerides among human lipoproteins. But the core cholesterol synthesis pathway has been considered largely conserved between humans and mice, and the mouse model has been widely used to study the regulation of human cholesterol synthesis genes (35). The impact of hLMR1, a nonconserved human lncRNA, on human cholesterol synthesis suggests that there might be additional layers of species-specific regulatory mechanisms beyond protein-coding genes in mammalian cholesterol metabolism. Consistent with this notion, a primate-specific lncRNA, CHROME, has also recently been shown to regulate cholesterol efflux in human macrophages and hepatocytes (36). These observations suggest that the regulation of the cholesterol synthesis pathway might also be subject to substantially divergent regulations among mammals, and defining the human IncRNAs involved could provide novel insights into human cholesterol metabolism.

The regulatory role of the hLMR1-PTBP1 complex identified in this study also provides new insights into the molecular basis specificity to PTBP1 function. Consistent with this notion, hLMR1 is highly specific to the liver, the primary site for cholesterol synthesis in the body. With the growing number of human lncRNAs shown to bind to PTBP1, studying the in vivo function of these lncRNAs is expected to enhance our understanding of the functional importance of PTBP1 in physiology and human diseases.

LncRNAs are the largest and probably also the least conserved transcript class in the human genome (15). With the growing importance of human lncRNAs in biology and physiology, defining hLMRs could drive profound changes in the way we study energy metabolism experimentally and understand metabolic disease conceptually, and we hope that efforts to define a functional hLMR could help chart the way forward.

\section{Methods}

\section{Bioinformatics analysis}

Analysis pipeline for human RNA-Seq data. FASTQ read files were cleaned using TrimGalore. Reads were then aligned using HISAT2 to an index created using the GRCh38 genome and the lncRNAKB annotation (23). Aligned.sam files were then compressed into. bam files and sorted using Sambamba sort. featureCounts from the subread package was used to count reads/fragments aligned to genes (at the exon feature level). Samples with less than 1,000,000 reads aligned were removed. Individual count files were merged into a single count file for each data set using a python script. In each data set, a threshold of more than $1 \mathrm{cpm}$ in at least $50 \%$ of 
samples was applied before further analysis. The RNA-Seq data sets for cross-sectional human studies were retrieved from BioProject PRJNA512027 for the 139 liver samples from the NAFLD population. The RNA-Seq data sets for interventional human studies were retrieved from BioProject PRJNA420975 for the 7 paired liver samples of the low-carbohydrate dietary intervention in NAFLD in the NCBI's Sequence Read Archive (SRA) database.

Analysis pipeline for humanized mice RNA-Seq data. RNA-Seq data have been deposited in NCBI's Gene Expression Omnibus (GEO, GSE130525). A combined human and mouse annotation was created by combining the lncRNAKB gene transfer format (GTF) annotation with the RefSeq GCF000001635.26 GTF annotation. A combined human and mouse genome was created by combining the GRCh38.p12 genome sequence; the primary assembly was obtained from the Gencode website and the GRCm38.p6 genomic sequence obtained from the RefSeq FTP site. RNA-Seq cleaning, alignment, sorting, quantification, and filtering were conducted in the same way as human RNASeq analysis using this index and genome file. Human gene counts were extracted from the resulting count file by removing reads mapped to mouse contigs. A more than $1 \mathrm{cpm}$ in $50 \%$ of samples cutoff was applied separately to human gene counts and mouse gene counts.

Differential expression analysis and principal component analysis. A combined raw count file generated by the subread featureCounts tool for each data set was imported into R. Technical replicates were combined using the collapseReplicates function from the DESeq2 package. The variance stabilizing transform from the DESeq2 package was applied to the count data from before and after combining technical replicates (if there were technical replicates) before conducting principal component analysis. The top 2 PCs were graphed to visualize clustering between experimental groups. DESeq2 was used with non-normalized count data to find differentially expressed genes between experimental groups. Covariates were controlled for by adding them to experimental design if available. A cutoff of logFC more than 0.4 and $q$ less than 0.05 was used for differential expression for all the liver samples.

Gene variability analysis. The gene expression profiles of the human liver from GTEx version 7 includes samples from Caucasian, Asian, and African ancestry (primarily Caucasian). An expression cutoff of more than $1 \mathrm{cpm}$ in $50 \%$ samples was applied in each tissue to reduce mapped genes to 16,906 expressed genes, including 2665 lncRNA genes in the liver. For each of the expressed genes, we quantified expression variability by calculating its coefficient of variation $\eta$ across all the available samples in each tissue. These were subsequently ranked and split into quartiles. Diseases category analysis was performed by using the DAVID gene functional annotation tool.

Gene correlation analysis. Count data for each data set was variance stabilizing transform (VST) normalized using the DESeq2 R package. After VST normalization, 10 hidden technical factors were calculated per data set using the probabilistic estimation of expression residuals (PEER) software package and were used in linear regression as covariates to correct VST normalized expression data. The mean expression of each gene was added back to the expression residual. The correlation between lncRNAs and protein-coding genes was analyzed by Pearson's method using the normalized data in the GTEx human population and metabolic disease-relevant population. Significantly correlated protein-coding genes with $P$ less than 0.05 in the liver were used for further $\mathrm{GO}$ analysis.

\section{Mice with a humanized liver}

TK-NOG mice, in which a herpes simplex virus type 1 thymidine kinase (TK) transgene under a mouse albumin promoter is expressed within the liver of highly immune-deficient NOG mice, were obtained from Taconic Biosciences. The TK converts an antiviral medication ganciclovir (GCV) into a toxic product that allows selective elimination of TK-positive cells in vivo. The cryopreserved primary human hepatocytes were obtained from Lonza. The humanized TK-NOG mice were prepared as previously described (29). Briefly, the TK-NOG mice at 8-9 weeks old received an i.p. injection of GCV at a dose of $25 \mathrm{mg} / \mathrm{kg}$. One week later, $50 \mu \mathrm{L}$ volume of $1 \times$ $10^{6}$ human primary hepatocytes suspended in HBSS solution were transplanted via intrasplenic injection. The serum human albumin in the mice was measured as an index of the extent of human hepatocyte replacement 8-12 weeks after transplantation. Humanized TK-NOG mice with serum human albumin levels above $0.5 \mathrm{mg} / \mathrm{mL}$ were used for experiments in which human hepatic genes could be reliably detected by qPCR. For the fasting-refeeding study, humanized mice were produced, and the experiment was carried out at Central Institute for Experimental Animals (CIEA). Humanized mice for the rest of the study were produced and analyzed at National Heart, Lung, and Blood Institute (NHLBI). For the fasting-refeeding study, humanized TK-NOG mice were allowed free access to food (fed, harvested at around 9-10 am) or subjected to a 24-hour food withdrawal (fasting, from 9:00 am to 9:00 am), or subjected to a 24-hour food withdrawal followed by a 4-hour refeeding (refeeding from 9:00 am to 1:00 pm) before tissue harvest. Animal data were excluded from experiments based on preestablished criteria of visible abnormal liver structure during sample harvest or other health issues such as fighting wounds or infections. According to the variability of metabolic parameters, group size was determined based on previous studies using similar assays within the laboratory and pilot experiments. Experimenters were not blinded to treatment group.

\section{RNA extraction, RNA-Seq, qPCR analysis}

Total RNA was isolated from liver tissues using TRIzol reagent (Invitrogen). After Turbo DNA-free DNase treatment (Ambion), the construction of strand-specific sequencing libraries using Illumina TruSeq RNA sample Prep kit and the sequencing were performed at the NHLBI DNA Sequencing and Genomics Core. The reverse transcription was carried out with SuperScript III First-Strand Synthesis system (Invitrogen) using $1 \mu$ g of RNA. Quantitative real-time RT-PCR was performed on a ViiA 7 Real-Time PCR System (Applied Biosystems Inc.) The PCR program was 2 minutes 30 seconds at $95^{\circ} \mathrm{C}$ for enzyme activation, 40 cycles of 15 seconds at $95^{\circ} \mathrm{C}$, and 1 minute at $60^{\circ} \mathrm{C}$. Melting curve analysis was performed to confirm the real-time PCR products. For detecting the expressions of human genes in humanized liver samples, human-specific primers were designed and quantitation was normalized to human 16S rRNA levels. For detecting the expressions of mouse genes in regular mice (C57BL/6), 18S rRNA was used as the internal control. For detecting the expression of hLMR1 in human tissues, the Human MTC Panel I (Takara Bio Inc., 636742) was used. The full primer sequences used are provided in Supplemental Figure 5.

\section{In vitro translation}

The in vitro translation analysis was performed using the TnT Quick Coupled Transcription/Translation System from Promega 
by following the manufacturer's protocol. The translated protein was visualized by using IRDye streptavidin (LI-COR), which detected the biotinylated lysine incorporated into the translated proteins. The open reading frame of yellow fluorescent protein (YFP) was used as a positive control.

\section{smRNA FISH}

smRNA FISH and microscopy were performed according to published protocols and as described previously $(44,45)$. Probe set against hLMR1 labeled with Quasar 570 was custom designed using LGC Biosearch Technologies' Stellaris online probe designer (version 4.2). GAPDH was hybridized with a predesigned probe set labeled with Quasar 670 (LGC Biosearch Technologies).

Primary human hepatocytes (Lonza) were seeded on collagen-coated glass coverslips (Thermo Fisher Scientific, NC0636242) in full growth media. The next day, coverslips were washed 2 times with PBS and fixed in 3.7\% formaldehyde (Sigma-Aldrich) in PBS for 10 minutes at room temperature (RT). After fixation, cells were permeabilized in $70 \%$ ethanol at $4^{\circ} \mathrm{C}$ for 1 hour. Coverslips were incubated in prehybridization buffer $(10 \%$ deionized formamide [Agilent] in wash buffer A [LGC Biosearch Technologies]) for 5 minutes at RT. Cells were hybridized with $50 \mu \mathrm{L}$ of hybridization buffer (LGC Biosearch Technologies) supplemented with 10\% deionized formamide (1:100 dilution of smRNA FISH probes) overnight at $37^{\circ} \mathrm{C}$ in a humid chamber. The next day, cells were washed with wash buffer (wash buffer A with 10\% deionized formamide) for 30 minutes at $37^{\circ} \mathrm{C}$, followed by a another wash (wash buffer A with $10 \%$ deionized formamide) containing Hoechst DNA stain (1:1000; Thermo Fisher Scientific) for 30 minutes at $37^{\circ} \mathrm{C}$. Coverslips were washed with wash buffer B (LGC Biosearch Technologies) for 5 minutes at RT, equilibrated for 5 minutes in base glucose buffer ( $2 \times$ SSC, $0.4 \%$ glucose solution, 20 mM Tris pH 8.0 in RNase-free $\mathrm{H}_{2} \mathrm{O}$ ), and then 5 minutes in base glucose buffer supplemented with 1:100 dilution of glucose oxidase (stock $3.7 \mathrm{mg} / \mathrm{mL}$ ) and catalase (stock $4 \mathrm{mg} / \mathrm{mL}$ ). The coverslips were mounted with ProlongGlass (Invitrogen) on a glass slide and left to curate overnight.

$Z$-stacks (250 nm z-step) capturing the entire cell volume were acquired with a GE wide-field DeltaVision Elite microscope with an Olympus UPlanSApo $100 \times / 1.40$ numerical aperture oil objective lens and a PCO Edge sCMOS camera using appropriate filters. The 3-dimensional stacks were deconvolved with the built-in DeltaVision SoftWoRx Imaging software. Fiji was used to generate maximum intensity projections and for signal quantification.

\section{Adenovirus production and in vivo adenovirus administration}

The shRNAs for hLMR1 and mouse Ptbp1 were designed using the following sequences (hLMR1 shRNA: CCTTCACAGCTCTGCCTAA; mouse Ptbp1 shRNA: GCACCGTCCTGAAGATCAT). The hairpin template oligonucleotides were synthesized by Integrated DNA Technologies and were subsequently cloned into the adenovirus vector of the pAD/Block-it system (Invitrogen) according to the manufacturer's protocols. The overexpression construct of hLMR1 was generated by PCR amplifying the sequence of ENST00000476385.1 using a human liver cDNA sample. The sequence was subsequently cloned into pAdv5 adenovirus vector for virus packaging. Adenoviruses were amplified in HEK293A cells and purified by $\mathrm{CsCl}$ gradient centrifugation. Purified viruses were desalted with PD10 columns (GE Healthcare Life Sciences) and titered with Adeno-X Rapid Titer Kit (Clontech). Adenoviruses were delivered into mice intravenously at $5 \times 10^{8} \mathrm{PFU} /$ mouse for overexpression experiments or at $1 \times 10^{9} \mathrm{PFU} /$ mouse for knockdown experiments. After 7 days, tissue samples were harvested for further analysis after mice had been fasted for 24 hours ( 9:00 am-9:00 am) and followed by a 4-hour refeeding ( 9:00 am to 1:00 pm).

\section{CRISPRa in humanized mice}

CRISPRa assay was conducted using the SAM system (http://sam. genome-engineering.org/protocols/). The sgRNA for hLMR1 was designed using the following sequence: forward, CACCGGACAGACAGGAGAGCAGACT; reverse, AAACAGTCTGCTCTCCTGTCTGTCC. Briefly, the template was ligated into the sgRNA (MS2) cloning backbone (Addgene, 61424) using Golden-Gate reaction (SAM) after Golden-Gate annealing. The expression cassettes of dCas9-VP64 (Addgene, 61422) and MS2-P65-HSF1 (Addgene, 61423) were subcloned into pAdv5 vector (Invitrogen) for virus packaging. SgRNA elements with the U6 promoter were amplified and subsequently cloned into pAd/PL adenovirus vector (Invitrogen) for virus packaging. Viruses were amplified, desalted, and titered as described above. Three adenoviruses (1:1:1) were delivered into humanized mice intravenously at a total of $5 \times 10^{8}$ $\mathrm{PFU} /$ mouse. After 7 days, tissue samples were harvested for further analysis after mice were under food withdrawal for around 5 hours (from 9:00 am to 2:00 pm).

\section{RNA pulldown assay}

RNA pulldown was performed as described previously (46). Briefly, biotin-labeled RNAs were transcribed in vitro using the Biotin RNA Labeling Mix and T7 RNA polymerase (Ambion) and purified with the RNeasy Mini Kit (QIAGEN). YFP coding sequence in a reverse direction (YFP-NC) was used as the control. Folded RNAs $(3 \mu \mathrm{g})$ were added into $5 \mathrm{mg}$ precleared liver lysates of humanized mice (supplemented with $0.2 \mathrm{mg} / \mathrm{mL}$ heparin, $0.2 \mathrm{mg} / \mathrm{mL}$ yeast tRNA, and $1 \mathrm{mM} \mathrm{DTT}$ ) and incubated at $4^{\circ} \mathrm{C}$ for 1 hour. Then 60 $\mu \mathrm{L}$ of washed streptavidin-coupled Dynabeads (Invitrogen) were added to each binding reaction and further incubated at $4^{\circ} \mathrm{C}$ for 1 hour. Beads were washed 5 times with lysis buffer $(150 \mathrm{mM} \mathrm{NaCl}$, 20 mM Tris pH 7.4, 1 mM EDTA, 0.5\% Triton X-100 with Protease/Phosphatase Inhibitor Cocktail and RNaseOUT, Thermo Fisher Scientific) and heated at $70^{\circ} \mathrm{C}$ for 10 minutes in $1 \times$ lithium dodecyl sulfate (LDS) loading buffer, and retrieved proteins were visualized by SDS-PAGE and silver staining. The unique protein bands shown in the hLMR1 RNA pulldown were identified by mass spectrometry analysis at NHLBI Proteomics Facility.

For RNA pulldown using the chromatin lysate, folded RNAs $(2 \mu \mathrm{g})$ were added into $5 \mu \mathrm{g}$ liver tissue chromatin lysate of regular mice (supplemented with $0.2 \mathrm{mg} / \mathrm{mL}$ yeast tRNA, $0.2 \mathrm{mg} / \mathrm{mL}$ DNA from salmon testes) and incubated at $4^{\circ} \mathrm{C}$ for 1 hour. Then, 30 $\mu \mathrm{L}$ of washed streptavidin-coupled Dynabeads (Invitrogen) were added to each binding reaction and further incubated at $4^{\circ} \mathrm{C}$ for 1 hour. Beads were washed 5 times with wash buffer $(150 \mathrm{mM} \mathrm{NaCl}$, 20 mM Tris pH 7.4, 1 mM EDTA, 0.5\% Triton X-100 with Protease/ Phosphatase Inhibitor Cocktail and RNaseOUT and $1 \mathrm{mM}$ DTT). The DNA was eluted by digesting the washed beads with RNase A and then treated with proteinase $\mathrm{K}$. The DNA was purified by using 
the column from Simple ChIP Enzymatic Chromatin IP kit (Cell Signaling Technology). The primers used were the same as used for mouse Ptbp1 ChIP analysis (Supplemental Figure 5).

\section{RIP analysis}

To prepare liver tissue lysates, frozen liver tissues were homogenized using a Dounce homogenizer with 15-20 strokes in RIP buffer $(150 \mathrm{mM} \mathrm{NaCl}, 20 \mathrm{mM}$ Tris pH 7.4, 1 mM EDTA, 0.5\% Triton $\mathrm{X}-100$ with Protease/Phosphatase Inhibitor Cocktail and RNaseOUT). For each RIP, $5 \mu \mathrm{g}$ rabbit IgG or PTBP1 antibody (32-4800, Thermo Fisher Scientific) were first incubated with $30 \mu \mathrm{L}$ washed Dynabeads Protein G in $300 \mu$ L RIP buffer supplemented with 0.2 $\mathrm{mg} / \mathrm{mL}$ BSA, $0.2 \mathrm{mg} / \mathrm{mL}$ heparin and $0.2 \mathrm{mg} / \mathrm{mL}$ EcoRI tRNA for 1 hour. Then, the antibody-coupled beads were added to $5 \mathrm{mg}$ of liver tissue lysates diluted in $500 \mu \mathrm{L}$ RIP buffer and incubated for 3 hours at $4^{\circ} \mathrm{C}$ with gentle rotation. Beads were washed briefly 5 times with RIP buffer. At the final wash, one-fifth of beads were used for protein analysis and the rest of the beads were resuspended in $1 \mathrm{~mL}$ of TRIzol for RNA extraction. Coprecipitated RNAs were isolated and analyzed by RT-PCR.

\section{ChIP analysis}

ChIP assays of frozen liver tissues of humanized mice were performed using the SimpleChIP Enzymatic Chromatin IP Kit (Cell Signaling Technology) according to the manufacturer's protocol.

IP was performed using RNA Poly II ChIP-validated antibody (MilliporeSigma, 17-620), PTBP1 antibody (Thermo Fisher Scientific, 32-4800), or with rabbit IgG as a negative control. For RNA poly II ChIP, the DNA in each ChIP were determined by qPCR analysis using primers amplifying the genomic sequences covering the transcriptional start sites of genes. For PTBP1 ChIP, the DNA in each ChIP were determined by qPCR analysis using primers amplifying the genomic sequences covering the promoters of genes. The primers used are list in Supplemental Figure 5. The relative enrichment was calculated by normalizing the amount of ChIP DNA to input DNA and comparing with the IgG control as fold enrichment.

\section{Luciferase reporter assay}

The human HMGCS1 promoter was amplified (forward, GTCCATCGGAATTAGTTTAGCCTGTGC; reverse, CAATCGCGGCCGGTAGAGTTG) and cloned into the pGL3-Basic Vector (Promega). Fulllength PTBP1 expression vector and control vector were purchased from OriGene (RC201779 and PS100001). The HEK293A cells were maintained in DMEM supplemented with $10 \%$ cosmic calf serum. Cells were transfected with pGL3-HMGCS1 promoter, the PTBP1, pAd-hLMR1, or control vectors using Lipofectamine 2000 (Invitrogen), and luciferase assays were performed 24 hours later using the Dual-Luciferase Reporter Assay Kit (Promega). Transfection efficiency was measured by normalization to Renilla luciferase activity expressed from a cotransfected pTK-RL vector (Promega).

\section{Immunoblotting}

For immunoblotting analyses, the cells and tissues were lysed in $1 \%$ SDS lysis buffer containing phosphatase inhibitors (Sigma-Aldrich) and a protease inhibitor cocktail (Roche). The lysate was subjected to SDS-PAGE, transferred to PVDF membranes, and incubated with the primary antibody followed by the fluorescence conjugated secondary antibody (LI-COR). The bound antibody was visualized using a quantitative fluorescence imaging system (LI-COR). The PTBP1 antibody (32-4800) was from Thermo Fisher Scientific.

\section{Measurement of lipid levels in liver tissues and plasma}

The liver and plasma cholesterol levels in regular mice were measured by using a cholesterol assay kit from Abcam (ab65390) and normalized to tissue weights. To measure human LDL-VLDL cholesterol levels in the plasma of humanized mice, human apolipoprotein $\mathrm{B}$-containing lipoproteins in the plasma of humanized mice were immunoprecipitated by using LipoSep IP reagent (Sun Diagnostics, LS-01). The immunoprecipitated pallets were first washed with PBS, and then were resuspended in PBS plus $0.5 \%$ NP-40 to release lipids from lipoproteins. After a brief centrifuge, the cholesterol levels in supernatant were measured by using a cholesterol assay kit from Abcam (ab65390) (as in Supplemental Figure 1D), or further normalized to the humanized ratio of each mouse (as in Figure 4D). The humanized ratio was determined by the relative expression levels of human $16 \mathrm{~S}$ in the real-time PCR analyses using cDNA prepared from the homogeneous powder of each humanized liver tissue.

\section{Statistics}

For comparisons between 2 groups, a 2-tailed, unpaired Student's $t$ test was used in the following figures: Figure 4, A and D, fasting versus fed, refeeding versus fasting; Figure 5, A-D; Figure 6, A and D; Figure 7, A-C; and Supplemental Figure 4, B and C. For multiple comparisons, 1-way ANOVA with post hoc Tukey's honestly significant difference test was used in Figure 6C. A P value of less than 0.05 was considered significant.

\section{Study approval}

All animal experiments were performed in accordance with and with approval from the NHLBI Animal Care and Use Committee or the Animal Care Committee of the CIEA, Kawasaki, Japan. All human-related data sets were downloaded from public domains.

\section{Author contributions}

$\mathrm{XR}, \mathrm{PL}, \mathrm{YM}, \mathrm{CJ}$, and HC designed the workflow. XR, PL, YM, and $\mathrm{CJ}$ performed most of the experiments with assistance from YS, NG, YO, NY, MN, GD, JLR, YH, KK, and HS. CJ, YC, PL, XR, FS, and MP performed bioinformatics analysis. XR, PL, YM, CJ, and $\mathrm{HC}$ wrote the manuscript. $\mathrm{HC}$ conceived and supervised the study. The order of the co-first authors is based on the length of time spent on and the contribution to the project.

\section{Acknowledgments}

We thank James Hawkins (NHLBI Animal Program) for assistance with the maintenance of humanized mice; Yan Luo, Poching Liu, and Yuesheng Li (NHLBI DNA Sequencing and Genomics Core) for RNA-Seq analysis; and Yong Chen and Marjan Gucek (NHLBI Proteomics Core) for their help with mass spectrometry analysis. We also thank Roy Parker and Carolyn Decker (University of Colorado Boulder) for access to the DeltaVision Elite microscope, and Theresa Nahreini and Nicole Kethley for the use of the Cell Culture Facility (University of Colorado Boulder). We are grateful to Michael N. Sack 
(NHLBI Cardiovascular Branch) for critical reading of the manuscript and helpful comments. The GTEx project was supported by the Common Fund of the Office of the Director of the NIH, and by NCI, NHGRI, NHLBI, NIDA, NIMH, and NINDS. NG was supported by the NIH Medical Research Scholars Program, a public-private partnership supported jointly by the NIH and contributions to the Foundation for the NIH from the Doris Duke Charitable Foundation (DDCF grant 2014194), Genentech, Elsevier, and other private donors. DDCF funds were not used to sponsor any of the research that involved animals. This study was funded by NHLBI Division of Intramural Research funds to HC (1ZIAHL006103, 1ZIAHL006159).

Address correspondence to: Haiming Cao, Cardiovascular Branch, National Heart, Lung and Blood Institute, National Institutes of Health, Bethesda, Maryland, USA. Phone: 301.402.9032; Email: haiming.cao@nih.gov. NG's present address is: Cleveland Clinic Foundation, Cleveland, Ohio, USA.
1. Zimmet P, Magliano D, Matsuzawa Y, Alberti G, Shaw J. The metabolic syndrome: a global public health problem and a new definition. J Atheroscler Thromb. 2005;12(6):295-300.

2. Swinburn BA, et al. The global obesity pandemic: shaped by global drivers and local environments. Lancet. 2011;378(9793):804-814.

3. Rosen ED, Spiegelman BM. Adipocytes as regulators of energy balance and glucose homeostasis. Nature. 2006;444(7121):847-853.

4. Hotamisligil GS. Inflammation and metabolic disorders. Nature. 2006;444(7121):860-867.

5 . Cao H. Adipocytokines in obesity and metabolic disease. JEndocrinol. 2014;220(2):T47-T59.

6. Iyer MK, et al. The landscape of long noncoding RNAs in the human transcriptome. Nat Genet. 2015;47(3):199-208.

7. Li P, et al. A liver-enriched long non-coding RNA, lncLSTR, regulates systemic lipid metabolism in mice. Cell Metab. 2015;21(3):455-467.

8. Ruan X, Li P, Cangelosi A, Yang L, Cao H. A long non-coding RNA, IncLGR, regulates hepatic glucokinase expression and glycogen storage during fasting. Cell Rep. 2016;14(8):1867-1875.

9. Zhao XY, et al. Long noncoding RNA licensing of obesity-linked hepatic lipogenesis and NAFLD pathogenesis. Nat Commun. 2018;9(1):2986.

10. Sallam T, et al. Feedback modulation of cholesterol metabolism by the lipid-responsive non-coding RNA LeXis. Nature. 2016;534(7605):124-128.

11. Sallam T, et al. Transcriptional regulation of macrophage cholesterol efflux and atherogenesis by a long noncoding RNA. Nat Med. 2018;24(3):304-312.

12. Yang L, et al. Integrative transcriptome analyses of metabolic responses in mice define pivotal lncRNA metabolic regulators. Cell Metab. 2016;24(4):627-639.

13. Lo KA, et al. Adipocyte long-noncoding RNA transcriptome analysis of obese mice identified Lnc-Leptin, which regulates leptin. Diabetes. 2018;67(6):1045-1056.

14. Uszczynska-Ratajczak B, Lagarde J, Frankish A, Guigó R, Johnson R. Towards a complete map of the human long non-coding RNA transcriptome. Nat Rev Genet. 2018;19(9):535-548.

15. Ulitsky I. Evolution to the rescue: using comparative genomics to understand long non-coding RNAs. Nat Rev Genet. 2016;17(10):601-614.

16. Necsulea A, et al. The evolution of IncRNA repertoires and expression patterns in tetrapods. Nature. 2014;505(7485):635-640.

17. Williams RB, Chan EK, Cowley MJ, Little PF. The influence of genetic variation on gene expression. Genome Res. 2007;17(12):1707-1716.

18. GTEx Consortium. Human genomics. The Gen-
otype-Tissue Expression (GTEx) pilot analysis: multitissue gene regulation in humans. Science. 2015;348(6235):648-660.

19. GTEx Consortium, et al. Genetic effects on gene expression across human tissues. Nature. 2017;550(7675):204-213.

20. Hagai $T$, et al. Gene expression variability across cells and species shapes innate immunity. Nature. 2018;563(7730):197-202.

21. Li J, Liu Y, Kim T, Min R, Zhang Z. Gene expression variability within and between human populations and implications toward disease susceptibility. PLoS Comput Biol. 2010;6(8):e1000910.

22. Simonovsky E, Schuster R, Yeger-Lotem E. Large-scale analysis of human gene expression variability associates highly variable drug targets with lower drug effectiveness and safety. Bioinformatics. 2019;35(17):3028-3037.

23. Seifuddin F, et al. IncRNAKB, a knowledgebase of tissue-specific functional annotation and trait association of long noncoding RNA. Sci Data. 2020;7(1):326.

24. Gerhard GS, Legendre C, Still CD, Chu X, Petrick A, DiStefano JK. Transcriptomic profiling of obesity-related nonalcoholic steatohepatitis reveals a core set of fibrosis-specific genes. J Endocr Soc. 2018;2(7):710-726.

25. Mardinoglu A, et al. An integrated understanding of the rapid metabolic benefits of a carbohydrate-restricted diet on hepatic steatosis in humans. Cell Metab. 2018;27(3):559-571.e5.

26. Guttman M, et al. Chromatin signature reveals over a thousand highly conserved large non-coding RNAs in mammals. Nature. 2009;458(7235):223-227.

27. Molyneaux BJ, et al. DeCoN: genome-wide analysis of in vivo transcriptional dynamics during pyramidal neuron fate selection in neocortex. Neuron. 2015;85(2):275-288.

28. Guttman M, Rinn JL. Modular regulatory principles of large non-coding RNAs. Nature. 2012;482(7385):339-346.

29. Hasegawa $M$, et al. The reconstituted 'humanized liver' in TK-NOG mice is mature and functional. Biochem Biophys Res Commun. 2011;405(3):405-410.

30. Ruan X, et al. In vivo functional analysis of non-conserved human IncRNAs associated with cardiometabolic traits. Nat Commun. 2020;11(1):45.

31. Lagarde J, et al. High-throughput annotation of full-length long noncoding RNAs with capture long-read sequencing. Nat Genet. 2017;49(12):1731-1740.

32. Gil G, Smith JR, Goldstein JL, Brown MS Optional exon in the 5'-untranslated region of 3-hydroxy-3-methylglutaryl coenzyme A synthase gene: conserved sequence and splicing pattern in humans and hamsters. Proc Natl Acad SciUS A. 1987;84(7):1863-1866.

33. Cookson W, Liang L, Abecasis G, Moffatt M, Lathrop M. Mapping complex disease traits with global gene expression. Nat Rev Genet. 2009;10(3):184-194.

34. Zhu Z, et al. Integration of summary data from GWAS and eQTL studies predicts complex trait gene targets. Nat Genet. 2016;48(5):481-487.

35. Shimano $\mathrm{H}$, et al. Elevated levels of SREBP-2 and cholesterol synthesis in livers of mice homozygous for a targeted disruption of the SREBP-1 gene. J Clin Invest. 1997;100 (8):2115-2124.

36. Hennessy EJ, et al. The long noncoding RNA CHROME regulates cholesterol homeostasis in primate. Nat Metab. 2019;1(1):98-110.

37. Huan L, et al. Hypoxia induced LUCAT1/PTBP1 axis modulates cancer cell viability and chemotherapy response. Mol Cancer. 2020;19(1):11.

38. Zhang L, Yang Z, Trottier J, Barbier O, Wang L. Long noncoding RNA MEG3 induces cholestatic liver injury by interaction with PTBP1 to facilitate shp mRNA decay. Hepatology. 2017;65(2):604-615.

39. Liu J, et al. Long non-coding RNA-dependent mechanism to regulate heme biosynthesis and erythrocyte development. Nat Commun. 2018;9(1):4386.

40. Lin N, et al. An evolutionarily conserved long noncoding RNA TUNA controls pluripotency and neural lineage commitment. Mol Cell. 2014;53(6):1005-1019.

41. Sun Z, et al. The long noncoding RNA Lncenc1 maintains naive states of mouse ESCs by promoting the glycolysis pathway. Stem Cell Reports. 2018;11(3):741-755.

42. Sasanuma H, Ozawa M, Yoshida N. RNA-binding protein Ptbp1 is essential for BCR-mediated antibody production. Int Immunol. 2019;31(3):157-166.

43. Senoo M, Takijiri T, Yoshida N, Ozawa M, Ikawa M. PTBP1 contributes to spermatogenesis through regulation of proliferation in spermatogonia. J Reprod Dev. 2019;65(1):37-46.

44. Raj A, van den Bogaard P, Rifkin SA, van Oudenaarden A, Tyagi S. Imaging individual mRNA molecules using multiple singly labeled probes. Nat Methods. 2008;5(10):877-879.

45. Dumbović G, et al. Nuclear compartmentalization of TERT mRNA and TUG1 lncRNA transcripts is driven by intron retention: implications for RNA-directed therapies. bioRxiv. doi: https:// doi.org/10.1101/2020.07.21.212514. Posted July 12, 2020. Accessed October 13, 2020.

46. Xing Z, Lin C, Yang L. LncRNA pulldown combined with mass spectrometry to identify the novel lncRNA-associated proteins. Methods $\mathrm{Mol}$ Biol. 2016;1402:1-9. 